\title{
The Complete DNA Sequence of the Mitochondrial Genome of a "Living Fossil," the Coelacanth (Latimeria chalumnae)
}

\author{
Rafael Zardoya and Axel Meyer
}

Department of Ecology and Evolution and Program in Genetics, State University of New York, Stony Brook, New York $11794-5245$

Manuscript received December 26, 1996

Accepted for publication April 14, 1997

\begin{abstract}
The complete nucleotide sequence of the 16,407-bp mitochondrial genome of the coelacanth (Latimeria chalumnae) was determined. The coelacanth mitochondrial genome order is identical to the consensus vertebrate gene order which is also found in all ray-finned fishes, the lungfish, and most tetrapods. Base composition and codon usage also conform to typical vertebrate patterns. The entire mitochondrial genome was PCR-amplified with 24 sets of primers that are expected to amplify homologous regions in other related vertebrate species. Analyses of the control region of the coelacanth mitochondrial genome revealed the existence of four 22-bp tandem repeats close to its $3^{\prime}$ end. The phylogenetic analyses of a large data set combining genes coding for rRNAs, tRNAs, and proteins (16,140 characters) confirmed the phylogenetic position of the coelacanth as a lobe-finned fish; it is more closely related to tetrapods than to ray-finned fishes. However, different phylogenetic methods applied to this largest available molecular data set were unable to resolve unambiguously the relationship of the coelacanth to the two other groups of extant lobe-finned fishes, the lungfishes and the tetrapods. Maximum parsimony favored a lungfish/coelacanth or a lungfish/tetrapod sistergroup relationship depending on which transversion:transition weighting is assumed. Neighbor-joining and maximum likelihood supported a lungfish/ tetrapod sistergroup relationship.
\end{abstract}

$\mathrm{C}$ OELACANTHS were for the first time recognized as a distinct taxonomic group $>150$ years ago by Louis Agassiz in his renowned book "Recherches sur les Poissons Fossiles" (1844). Since then, many more extinct coelacanth species have been discovered (ClOutIER and FOREY 1991). These fossils range in age from the Early Devonian to the Late Cretaceous (HuXLeY 1861; WOODWARD 1891; STENSIŌ 1921). No fossil coelacanths are known from more recent times, and, hence, this group was believed to have gone extinct $\sim 70-80$ mya. However, in 1938, a living coelacanth (Latimeria chalumnae) was trawled off the estuary of the Chalumna river, near East London, South Africa (SмiтH 1939, 1956; COURTENAY-Latimer 1979). Since the sensational discovery of this living fossil, a total of only $\sim 200$ specimens have been caught off the Comoro archipelago near the eastern coast of Africa in the Indian ocean. It proved to be the only known coelacanth population (SCHLIEWEN et al. 1993; Fricke et al. 1995).

Coelacanths are large gray-blue marine fishes whose morphology has remained seemingly unchanged during the last 400 million years (but see FOREY 1991). During the last 50 years, detailed anatomic and functional morphological analysis of the circulatory, reproductive, locomotive, and respiratory systems of the coelacanth have been conducted (e.g., MiLlot 1954, 1955;

Corresponding author: Rafael Zardoya, Department of Ecology and Evolution, 662 Life Sciences, State University of New York, Stony Brook, New York 11794-5245. E-mail: rzardoya@life.bio.sunysb.edu
SMITH 1956; ThOMSON 1966; Bemis and NorthCUTT 1991; Mangum 1991; Schultze and Cloutier 1991; TAMAI $e t a l$. 1994). These fishes are characterized by the possession of several distinct morphological, physiological, and life history traits, such as lobed paired fins, an heterocercal tail with an apical lobe, an intracranial joint, a rostral electroceptor organ, a urea retention mechanism and very large eggs (and give birth to living young) (e.g., FOREY 1988; LONG 1995; MAISEY 1996). Unlike other sarcopterygians, the group to which they belong (see below), coelacanths lack choanae (internal nostril), maxillae and functional lungs.

Unfortunately, little is known about the coelacanth at a molecular level. To date, only the triosephosphate isomerase (KolB et al. 1974), the $\alpha$ and $\beta$ parvalbumin (PECHERE et al. 1978), and the $\alpha$ and $\beta$ hemoglobin amino acid sequences (GORR et al. 1991), as well as the 18S rRNA (STOCK et al. 1991), 28S rRNA (Hillis et al. 1991; ZARDOYA and MEYER 1996b), cytochrome $b$ (MEYER and WILSON 1990), 12S rRNA (MEYER and WILSON 1990; MEYER and DOLVEN 1992; HEDGES et al. 1993), 16S rRNA (HEDGES et al. 1993), cytochrome oxidase subunit 1 (YOKOBORI et al. 1994), and Mhc class I (BETZ et al. 1994) nucleotide sequences have been reported from the coelacanth (reviewed by MEYER 1995).

Despite much prior effort, the phylogenetic position of the coelacanth still remains controversial (reviewed by MEYER 1995). It is well established that the coelacanth, together with lungfishes, and the extinct rhipidistians form the subclass Sarcopterygii, the lobe- 
TABLE 1

PCR and sequencing primers used in the analysis of the coelacanth mitochondrial genome

\begin{tabular}{|c|c|c|}
\hline PCR primers & Sequence $\left(5^{\prime} \rightarrow 3^{\prime}\right)$ & $\begin{array}{l}\text { Approximate product } \\
\text { length (bp) }\end{array}$ \\
\hline 1. $16 \mathrm{~S} \mathrm{~F}$ & AGT TCA GAC CGG AGC AAT CCA GG & 600 \\
\hline 2. LATI ND1 R & ACT TCG TAG GAA ATA GTT TGT GC & \\
\hline 3. LATI ND1 F1 & CCT TGA TCG GGG CCC TTC GAG CA & 600 \\
\hline 4. LATI MET R & TCG GGG TAT GGG CCC GAA AGC TT & \\
\hline 5. LATI GLN F & TGA ACC TAT ACT AAA GAG ATC AA & 750 \\
\hline 6. LATI ND2 R & ATT TTT CGT AGC TGC GTT TGA TT & \\
\hline 7. LATI ND2 F & GGA CTT ATC CTG TCG ACT TGA CA & 700 \\
\hline 8. LAATI TRP R & TTA AAG CTT TGA AGG CTC TTA GT & \\
\hline 9. LATI TRP F & AAG CAT CAT AAC CCT CAT AGC ACT & 700 \\
\hline 10. LATI COI R & GGC ATC ACT ATA AAG AAG ATT AT & \\
\hline 11. LATI COI F1 & GAT GAC CAA ATT TAT AAT GTA GT & 700 \\
\hline 12. LATI COI RI & ATT GCC ATT ATC GCT CAG ACT AT & \\
\hline 13. LATI COI F2 & AAG AAA GAA CCA TTC GGG TAT AT & 1200 \\
\hline 14. $\mathrm{H} 7886^{a}$ & TAN SWY CAR TAY CAY TGR TGN CC & \\
\hline 15. LATI COII F & TAA TTG ATG AAG TCG AAA ACC CTC A & 650 \\
\hline 16. LATI AT8 R & TTA GGC TCA TGG TCA GGT TCA & \\
\hline 17. LATI AT8 F & AGT GAA TGC CTC ARY TAA AYC C & 1000 \\
\hline 18. LATI COIII R & TTT TGT ACA GGT AGT GTG TGG TG & \\
\hline 19. LATI COIII F & ATA TAT CAA TGA TGA CGA GA & 600 \\
\hline 20. LATI COIII R1 & ACA TCA ACG AAA TGT CAG TAC CA & \\
\hline 21. LATI COIII F1 & TAC CAC TTC ACA TCA AAC CAC CA & 1600 \\
\hline 22. LATI ND4 R & TAG TAG GAC GGC GGC TAG TAC TAT & \\
\hline 23. LATI ND4 F1 & CCT AAR GCC CAY GTA GAR GC & 900 \\
\hline 24. LATI LEU R & TTT GCA CCA AGA GTT TTT GGT TCC TA & \\
\hline 25. LATI LEU F & CTA AAG GAT AAT AGC TCA TCC ATT & 600 \\
\hline 26. LATI ND5 R & CAY CAG CCR ATT ART ARR AAT GAY AT & \\
\hline 27. LATI ND5 F & CAR YTA TTY ATC GGN TGR GAR GG & 650 \\
\hline 28. LATI ND5 R1 & CCY ATY TTT CKG ATR TCY TGY TC & \\
\hline 29. LATI ND5 F2 & TAA AGC AAT GCT GTT CCT ATG CT & 850 \\
\hline 30. LATI ND5 R3 & AAN AGN GTN AGR TAN GTY TTR AT & \\
\hline 31. LATI ND5 F3 & ATC AAC AAC TCG CAA CAA GGA C & 1100 \\
\hline 32. $\mathrm{H} 15149^{b}$ & AAA CTG CAG CCC CTC AGA ATG ATA TTT GTC CTC A & \\
\hline 33. LATI CYTB F & TAC TTA CAA AAA GAA ACC TG & 850 \\
\hline 34. LATI THR R & CGG CTT ACA AGG GCC GAT GCT TT & \\
\hline 35. LATI THR-F & AGC CTT AGT AGC TTA AAC CC & 1000 \\
\hline 36. LATI12S-R & AGC AAG GCT GGG ACC AAA CCT TT & \\
\hline 37. L-Phe & TAA AGC ATA GCA CTG AAA ATG & 850 \\
\hline 38. $12 \mathrm{Sb}$ & GAG GGT GAC GGG CGG TGT GT & \\
\hline 39. 12Sa & TGG GAT TAG ATA CCC CAC TAT & 800 \\
\hline 40. $16 \mathrm{~S}-1 \mathrm{H}$ & KTA GCT CRC CYA GTT TCG GG & \\
\hline 41. 16S-1L & AGT ACC GCA AGG GAA ARC TGA AA & 800 \\
\hline 42. $16 \mathrm{~S}-2 \mathrm{H}$ & GAT TRY GCT ACC TTY GCA CGG TCA & \\
\hline 43. 16 Sar $^{\prime}$ & CGC CTG TTT ATC AAA AAC AT & 500 \\
\hline 44. $16 \mathrm{Sbr}^{\circ}$ & CCG GTC TGA ACT CAG ATC ACG T & \\
\hline
\end{tabular}


TABLE 1

Continued

\begin{tabular}{llc}
\hline \multicolumn{1}{c}{ PCR primers } & \multicolumn{1}{c}{ Sequence $\left(5^{\prime} \rightarrow 3^{\prime}\right)$} & $\begin{array}{c}\text { Approximate product } \\
\text { length }(\mathrm{bp})\end{array}$ \\
\hline 45. LATI ND6-F & ATC CCC CCY AAA TAR ATC AAA AA & 650 \\
32. H15149 & AAA CTG CAG CCC CTC AGA ATG ATA TTT GTC CTC A & \\
46. LATI 16S-F & CAA TCC TTT CCA AGA GTC CAA AT & 450 \\
47. LATI 16S-R & GTA CTG GCT CCT TAA TGA ATA GT & \\
Sequencing & & \\
LATI COI F3 & CCC TGC TAT GAG CAC TAG GAT TT & \\
LATI ND3 F & AAT GTG GCT TTG ATC CTC TAG GA & \\
LATI ND3 R & TTT AGT CGC TCT GTT TGA TTA CC & \\
\hline IUB code: $R$, A/G; Y, C/T; W, A/T; S, G/C; and K, G/T. & \\
&
\end{tabular}

finned fishes (Romer 1966; CARroll 1988; AHLBERG 1991; Cloutier and Ahlberg 1996) from which tetrapods were originated. The origin of land vertebrates from water-bound lobe-finned fishes involved both morphological and physiological innovations and was one of the most significant events in the history of vertebrates (PANCHEN and SMITHSON 1987). There is recent strong evidence supporting elpistostegids (or panderichthyids), a group of rhipidistians, as the extinct sister group of the earliest tetrapods (VOROBYEVA and Schultze 1991; Clack 1994; Schultze 1994; AHLberG et al. 1996; Cloutier and AHLberg 1996).

However, there is no general agreement about the relationships among the coelacanth, lungfishes and the rhipidistian+tetrapod clade (e.g., ScHultze 1994; for review, see MEYER 1995). Since molecular phylogenetic data can only be collected from extant species, they can contribute only in limited ways to this debate (MEYER and WILSON 1990; MEYER and DOLVEN 1992; HedGes $e t$ al. 1993; YOKOBORI et al. 1994; ZARDOYA and MEYER 1996b; see MEYER 1995 for a review). However, the establishment of the phylogenetic relationships among the three extant sarcopterygian groups, i.e., lungfishes, the coelacanth and tetrapods, can be accomplished by the evolutionary analyses of molecular data sets and would help to recreate the sequence of evolutionary events that might have preadapted for the extinct sarcopterygian conquest of land. All three possible competing hypotheses that explain the relationships among living representatives of sarcopterygians, have been supported at various times by either morphological and/ or molecular data: (1) the lungfishes as the closest living relatives of tetrapods (ROSEN et al. 1981; FOREY 1987, 1988; MEYER and WILSON 1990). (2) The coelacanth as the sister group of tetrapods among living vertebrates (FrITZSCH 1987; SCHUlTZE 1987; GorR et al. 1991). And (3) lungfishes and the coelacanth form a monophyletic group and, hence, are equally closely related to tetrapods (NorthCutT 1987; Forey 1988; Chang 1991; Schultze 1994; ZardoyA and MeYer 1996b). The apparently rapid successive origin and subsequent diversification of the three remaining lineages of sarcopterygians back in the Devonian makes the determination of their phylogenetic relationships difficult. Many mor-

\section{Latimeria chalumnae mtDNA}

$$
16,407 \text { bp }
$$

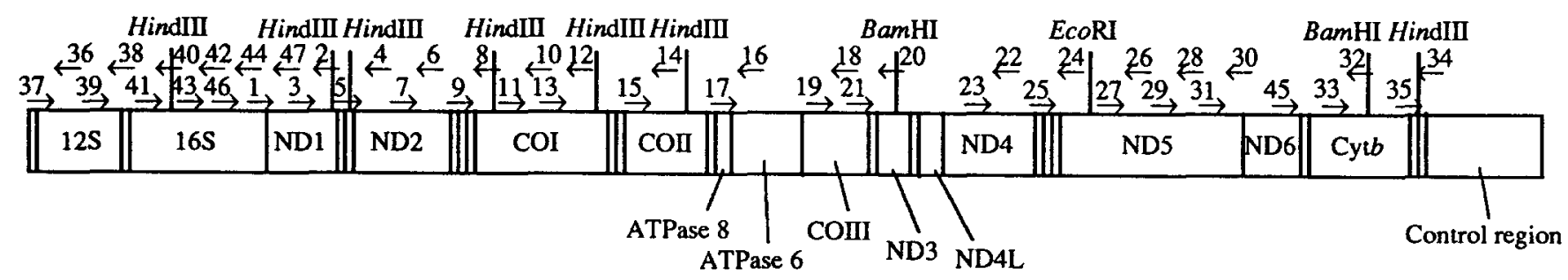

FIGURE 1.-Gene organization, restriction map, and cloning/sequencing strategy for the coelacanth mitochondrial genome. All protein coding genes are encoded by the $\mathrm{H}$ strand with the exception of ND6, which is coded by the $\mathrm{L}$ strand. Localization and direction of the primers used in the PCR amplification are denoted by arrows (see Table 1 for the primer DNA sequence associated with each number). 
tRNA-Phe $\rightarrow \quad-\quad 125$ rRNA $\rightarrow$

1 GTGAGTGTAGCTTAATAAAAAGCATAGCACTGAAGATgCTAAGATGAATTACAAAATATTCCACTGACACAAAGgTTTGGTCCCAGCCTTGCTATCAATT

101 TTAACCAGGATTACACATGCAAGCATCAACTCCCCAGTGAGAATGCCCCTGACTTATCCGTCAAAGATAACAGgGAGTAGGTATCAGGCACACAACATTA

ACGCTAGCCCAAGACACCTTGTCCAGCCACACCCCCAAGGGAACTCAGCAGTGATAGACATTGAATAATAAGTGAAAACTTGACTCAGCCATGGTTACAA

GGGCCGGTCAACTCCGTGCCAGCCACCGCGGTTACACGGAAGACCCAAAATGATAACACTACCGGCGTAAAGCGTGATTAAAGGACACCCACCATAATGG

AGCCACAAATAACTAAAGCTGTTATACGCACTTAAAAAAATATGCTCATCACACGAAAGTAACTCCAGCACCCAAAGGAACCCTGAACCCACGAAAGCTA

AGAAACAAACTGGGATTAGATACCCCACTATGCTCAGCCCTAAACACAAACAATTCAAACACACACTGTTCGCCAGGGGAACTACAAGCGCCAGCTTCAA

TATATACCGCCGTCGCCAGCCCACCCTGTGAAGGAAATACAATGGGCAAAAATAAAAAAATTAAAAACGTCAGGTCGAGGTGTAGCAAATGAGATGGGAA

GAAATGGGCTACATTTTCTAAATATAGAATATTACGAAAAAATACAGCGAAACCTGTACTTTGAAGGAGGATTTAGCAGTAAAAGgGGAaTAGAGAGCCC

CTCTGAAACCGGCCTGAAATGCGCACACACCGCCCGTCACTCTCCTCACCCAAAATCGGCCCCATCTTTTAATAAACAAAAAACCAAGCATACAAGTAG

tRNA-Val $\rightarrow$

AGGAGGCAAGTCGTAACAAGGTAAGTGTACCGGAAGGTGCACTTGGACTAATCAAAATETAGTTTAACAAAACACCTCCCMTACACTGAGAAGACACCCA $16 \mathrm{~S}$ IRNA $\rightarrow$

1101 GGAAAGGGGTCATTTTGAGCTATATAGCTAGgCCAACAAAACCATAACCACTACACCATTAACCAAAAACTTGAACAAACAAAACCTAAACCATTTACC

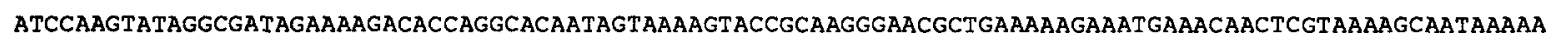

CCAAAGACTAACCCTTGTACCTTTTGCATTATGATCTAGTTAGACCCCACCGGGCAAAATGAATTTAAGTCCAACCCCCCGAAACTAAGTGAGCTACTTC

GAAACAGCCTATGAGGGCAAACCCTTCTCTGTGGCAAAAGAGTGGGAAGATTTCCAAGTAGAGGCGATAAACCTAACGAGCTTAGTGATAGCTGGTTATT GCTTAAGCAGCACTAAACCAACCTATACTAATAAATTTAACCTCATTCCACCATCACTATCGAATTATTCTATATGCATAGAAGAATAAATGCTAGAATT AGTAACAAGAAGgCCATTAAGCCTTCTCTAACTGCATAAGTGTACATCAGATTAGATTAACCACTGATAATTAACGACTTCAAAGAGAATACTATGACAT

AAAACAAGAAAAGCACACACGCCCACATCGTTAATCCAACACAGGAATGCAACCACGAAAGATTAAAAGAAAGAAAAGGAACTCGGCAAACTATAAGCCC CGCCTGTTTACCAAAAACATCGCCTCCCGCCAACAACAGAAGTATTGGAGGTCCCGCCTGCCCAGTGACAAGATTTAACGGCCGCGGTATCCTGACCGTG CAAAGGTAGCGAAATCACTTGTCTTTTAAATGAAGACCTGTATGAATGGCACCACGAGGGCTTAACTGTCTCCTCTTTCCAATCAGTAAAATTGATCTGT

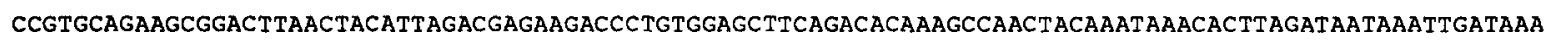
ACCAGTAGCCTAATACTGGCCCTATTGTCITTGGTTGGGGCGACCACGGAGAAAAAACAATCCTCCAAGCCGATTGGTACCACCTGTACTAAAACAAAGG GTAACACCCCAAAGTAATAAAAATTTTATCGGACATGACCCAGGGACTAAACCTGATCAACGAACCAAGTTACCCCAGGGATAACAGCGCAATCCTTTCC AAGAGTCCAAATCGACGAAAGGGTTACGACCTCGATGTTGGATCAGGACACCCCAATGGTGAAGCCGCTATTAAAGGTTTGTTTGTTCAACAATTAAAG TCCTACGTGATCTGAGTTCAGACCGGAGAAATCCAGGTCAGTTTCTATCTATGATGTTAATTCTCCCAGTACGAAAGGACCGGAGAACTTGAGCCAATGC

2701 CACAAGCACGCTCATCTTCAACCTGATGAAAACAACTAAAACAGATAAAGAAGAACACAACCGCTCCCGTAAACAACGGGCAATGCCGGGGTGGCAGAGC $\mathrm{NADH} I \rightarrow$

$\begin{array}{llllllllllllllllll}M & T & K & I & I & T & H & L & L & N & P & L & A & V\end{array}$

2801 CTGGCTAAATGCAAGAGGCCTAAACTCTCTCTCTCAGAAGTTCAAATCCTCTCCCCAGCTATGACAAAAATTATTACACACCTGCTAAACCCACTAGCTG

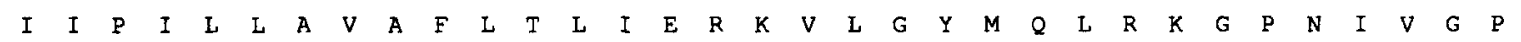

2901 TCATCATTCCAATTTTACTAGCCGTAGCGTTCCTGACACTCATCGAACGAAAAGTACTAGGTTATATGCAACTACGAAAGGGTCCTAATATCGTAGGCCC $\begin{array}{lllllllllllllllllllllllllllllllllllll}Y & G & L & L & Q & P & L & A & D & G & L & K & L & F & I & K & E & P & V & R & P & S & T & S & S & P & L & L & F & I & T & T & P\end{array}$

3001 ATATGGTCTCCTACAACCCCTAGCAGATGGACTAAAACTATTCATTAAGGAGCCAGTACGACCTTCTACATCATCCCCGCTACTCTTCATTACCACTCCA

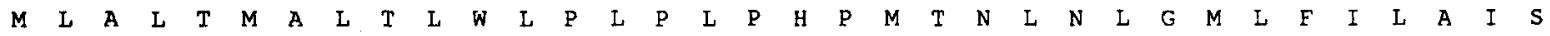
3101 ATACTAGCACTCACCATAGCACTAACTCTATGACTACCACTACCCCTCCCACATCCAATAACAAACCTAAACCTAGGAATATTATTTATCCTAGCAATCT $\begin{array}{llllllllllllllllllllllllllllllllllllllll}S & L & T & V & Y & S & I & L & G & S & G & W & A & S & N & L & K & Y & A & L & I & G & A & L & R & A & V & A & Q & T & I & S & Y\end{array}$ 3201 CAAGTCTAACAGTATACTCAATTCTAGGCTCCGGCTGAGCATCAAACTTAAAATATGCCCTAATTGGGGCCCTCCGAGCAGTCGCACAAACAATCTCCTA

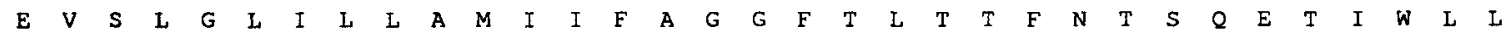

3301 TGAAGTAAGCCTAGGACTTATCCTACTGGCCATAATCATCTTCGCAGGCGGTTTTACACTAACAACATTTAATACATCACAGGAAACCATTTGACTTCTA

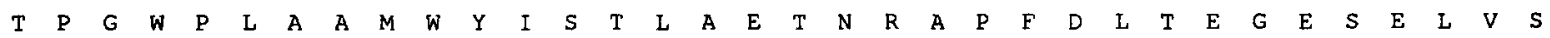
3401 ACGCCAGGATGACCACTCGCAGCAATATGATACATCTCAACCTTAGCAGAAACCAACCGAGCCCCATTTGACCTCACAGAAGGAGAATCAGAACTTGTAT

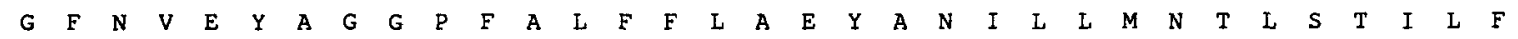
3501 CGgGGTTPAATGTGGAATATGCAGGAGGACCATTTGCACTATTCTTTCTAGCAGAATATGCAAATATTCTACTAATAAACACACTATCAACTATTCTATT

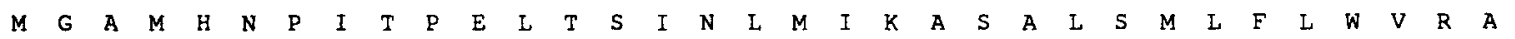

3601 TATAGgaGCCATACACAACCCAATCACACCAGAaCTAACCTCAATTAACCTAATGATTAAAGCCTCCGCACTATCAATACTCTTCCTATGAGTACGAGCC

FIGURE 2.-Complete L-strand nucleotide sequence of the coelacanth mitochondrial genome. Position 1 corresponds to the first nucleotide of the $t R N A^{\text {Phe }}$ gene. Direction of transcription for each gene is represented by arrows. The deduced amino acid sequence for each gene product is shown above the nucleotide sequence (one-letter amino acid abbreviation is placed above the first nucleotide of each codon). Complete termination codons are indicated $(*)$. tRNA genes are underlined and the corresponding anticodons are overlined. In the control region, four repeats are shown. 
$\begin{array}{lllllllllllllllllllllllllllllllllllll}S & Y & P & R & F & R & Y & D & Q & L & M & H & L & V & W & K & N & F & L & P & I & T & L & A & M & I & L & W & H & T & S & L & P & I\end{array}$ 3701 TCATACCCACGATTCCGATATGACCAGCTAATACACTTAGTATGAAAAAACTTCTTACCAATCACCCTAGCCATGATCCTATGACACACCTCCCTTCCAA $F \quad T \quad G \quad S \quad L \quad P \quad P \quad Q \quad T$ * tRNA-Ile $\rightarrow$

3801 TTTTTACAGGAAGCTTACCACCACAAACCTAAAGGAAACGTGCCCGAATAAACAAGgACCACTTTGATAGAGTGGACTATAGGAGTTAAAACCTCCTCGC $\leftarrow$ tRNA-GIn

tRNA-Met $\rightarrow$

3901 TTCCTAGGAAAATAGGACTTGAACCTATACTAAAGAGATCAAAACTCTTGGTGCTACCACTACACCATCTCCTAGTAAAGTCAGCTAAAAAAGCTTTCGG NADH $2 \rightarrow$

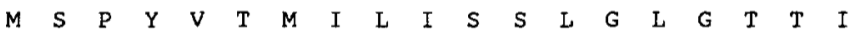

4001 GCCCATACCCCGAACATGTTGGTTAAACCTCCCTCCTTTACTAATGAGCCCTTACGTAACAATAATCCTTATCTCAAGCCTTGGACTCGGGACAACAATI $\begin{array}{lllllllllllllllllllllllllllllllllllll}T & F & T & S & S & S & W & L & M & A & W & M & G & L & E & I & N & T & L & A & I & T & P & L & M & V & K & Q & H & H & P & R & A & T\end{array}$ 4101 ACATTIACAAGCTCATCCTGACTGATAGCTTGAATAGgTCTAGAAATTAATACCCTAGCCATCACCCCCCTAATAGTAAAACAACATCACCCTCGGGCAA $\begin{array}{llllllllllllllllllllllllllllllllll}E & A & T & T & K & Y & F & L & T & Q & A & T & A & S & G & L & L & L & F & A & T & L & N & N & A & W & M & T & G & E & W & N & T\end{array}$ 4201 CTGAAGCCACAACAAAATATTTTCTTACCCAGGCAACAGCATCAGGACTGCTATTATTCGCAACCCTTAACAACGCTTGGATAACAGGAGAATGAAACAC $\begin{array}{lllllllllllllllllllllllllllllllllll}M & E & L & S & N & N & L & S & A & P & M & I & T & M & A & L & A & L & K & M & G & V & A & P & M & \text { H } & F & W & L & P & E & V & L\end{array}$

4301 AATAGAACTATCAAACAATTTATCCGCCCCAATAATTACAATAGCCCTCGCACTAAAGATAGGAGTAGCACCAATACACTTCTGATTACCAGAAGTGCTA $\begin{array}{lllllllllllllllllllllllllllllllllll}Q & G & L & P & L & L & T & G & L & I & L & S & T & W & Q & K & L & A & P & F & T & L & L & Y & M & T & S & H & E & L & N & T & T & T\end{array}$ 4401 CAAGGACTCCCCCTACTAACTGGACTTATCCTGTCGACTTGACAAAAACTAGCCCCCTTCACCCTACTATATATAACATCACATGAATTAAACACAACAA $\begin{array}{llllllllllllllllllllllllllllllllll}M & T & I & L & G & L & T & S & T & I & I & G & G & L & G & G & L & N & Q & T & Q & L & R & K & V & L & A & Y & S & S & I & A & H\end{array}$ 4501 CAATAACAATCCTAGGATTGACATCAACAATTATCGGTGGCCTTGGTGGATTAAACCAAACTCAACTGCGAAAAGTCCTAGCTTACTCATCAATTGCACA $\begin{array}{lllllllllllllllllllllllllllllllllll}L & G & W & M & V & I & I & I & Q & Y & S & K & T & L & A & L & L & N & L & L & L & Y & I & T & M & T & S & T & A & F & L & T & L\end{array}$

4601 CCTCGGATGAATAGTTATCATTATCCAATACTCCAAAACACTAGCCCTACTAAACCTACTGCTATATATTACAATAACATCAACAGCATTTTTAACACTT $\begin{array}{lllllllllllllllllllllllllllllllllllllllll}M & T & L & S & A & T & K & I & N & T & L & S & T & K & W & A & T & T & P & I & A & I & M & T & A & M & L & A & L & L & A & L & G & G\end{array}$

4701 ATGACTCTATCAGCCACAAAAATTAATACCCTATCAACAAAATGAGCAACAACCCCTATCGCAACTATAACTGCAATACTAGCTCTACTAGCATTAGGAG $\begin{array}{lllllllllllllllllllllllllllllllllll}L & P & P & L & T & G & F & M & P & K & W & L & I & L & Q & E & L & T & K & Q & N & L & P & A & L & A & T & L & M & A & L & S & A\end{array}$

4801 GTCTCCCACCACTAACAGGATTTATACCAAAATGACTAATTTTACAAGAACTTACCAAGCAAAATTTACCCGCTCTAGCCACACTAATAGCCCTATCAGC $\begin{array}{lllllllllllllllllllllllllllllllllllll}L & L & S & L & F & F & Y & L & R & M & C & H & T & M & T & L & T & I & S & P & N & T & N & N & N & M & I & T & W & R & K & K & P\end{array}$

4901 CCTATTAAGCCTCTTCTTCTACCTACGAATATGCCATACAATAACCCTTACAATCTCACCAAACACAAACAATAACATAATTACATGACGAAAGAAACCT $\begin{array}{llllllllllllllllllllllllllllllllllllll}G & Q & K & A & L & P & L & A & M & L & S & I & M & T & L & M & A & L & P & T & T & P & T & M & V & A & I & M & N & * & \text { tRNA-TrP }\end{array}$

5001 GGCCAGAAAGCACTACCCCTAGCCATACTAAGCATCATAACCCTCATAGCACTCCCAACAACCCCAACAATAGTAGCCATCATAAACTAATAAGAGACTI $\rightarrow$

5101 AGGAATAAACCAAGACCAAGAGCCTTCAAAGCCCTCAGCAGGAGTGAAAACC'TCCTAGTCTCTGGATAAGACCTGTAGGATACTACCCCACATATTCTGA $\leftarrow$ tRNA-Ala

5201 ATGCAAACCAGACACTTTAATTAAGCTAAGGCCTCCACTAGATGGGLAGGCCTCGATCCTACAAAATATTAGTTAACAGCTAAAAGCCCAAACCGGCGAG $\leftarrow$ tRNA-Asn $\leftarrow$ tRNA-

5301 CATCCATCCTAACATTCCAAGAAAAAAAGGAATGTT TGAAGCCTCAACAAGCACCACTTGTCCCTCCAGGTTTGCAACCTGACATECTAAACACCATGAG $\mathrm{COI} \rightarrow$

Cys

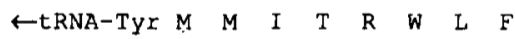

5401 GCTTGGTAAGAGGAGGAATTGAACCTCCCTACACGGGGCTACAACCCGCCGCTAAGAGCAATCAGCCATCTTACCTGTGATAATCACTCGTTGACTATTC $\begin{array}{lllllllllllllllllllllllllllllllllllllllllll}S & T & N & H & K & D & I & G & T & L & Y & M & I & F & G & A & W & A & G & M & V & G & T & A & L & S & L & L & I & R & A & E & L & S\end{array}$

5501 TCAACCAACCATAAAGACATTGGTACCCTATACATGATCTTCGGTGCCTGAGCTGGAATAGTTGGAACCGCCCTAAGCCTGCTTATTCGAGCTGAACTCA $\begin{array}{llllllllllllllllllllllllllllllllllll}Q & \text { P } & G & \text { A } & \text { L } & \text { L } & G & \text { D } & \text { D } & Q & \text { C } & \text { I } & \text { Y } & \text { N } & \text { V } & \text { V } & \text { V } & \text { T } & \text { A } & \text { H } & \text { A } & F & \text { V } & M & \text { I } & F & F & M & V & M & P & I & M & I\end{array}$ 5601 GCCAACCTGGGGCTCTCCTGGGCGATGACCAAATTTATAATGTAGTCGTTACAGCACATGCATTCGTGATAATCTTCTTTATAGTAATACCGATCATAAT $\begin{array}{llllllllllllllllllllllllllllllllllllll}G & G & F & G & N & W & L & I & P & L & M & I & G & A & P & D & M & A & F & P & R & M & N & N & M & S & F & W & L & L & P & P & S\end{array}$

5701 CGGCGGGTTTGGCAACTGATTAATTCCCCTGATGATTGGGGCACCCGACATAGCATTTCCACGTATAAACAACATAAGCTTCTGACTACTACCACCCTCA

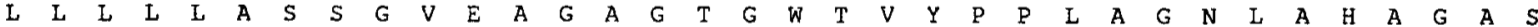

5801 CTCCTACTCCTACTAGCATCTTCTGGAGTAGAAGCAGGAGCAGGCACAGGATGGACAGTATACCCTCCACTAGCGGGCAACCTCGCCCATGCAGGAGCAT $\begin{array}{lllllllllllllllllllllllllllllllllllll}V & D & L & T & I & F & S & L & H & L & A & G & V & S & S & I & L & G & A & I & N & F & I & T & T & V & I & N & M & K & P & P & T\end{array}$ 5901 CCGTAGATTTAACAATITICTCCTTACATCTAGCCGGTGTATCCTCAATCTTAGGGGCCATCAACTTCATCACAACAGTAATCAACATAAAACCCCCAAC

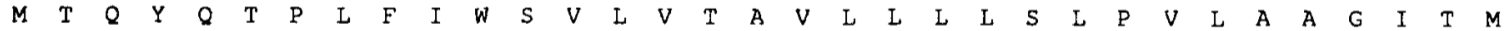

6001 AATAACACAGTATCAGACACCACTATTTATCTGATCAGTCTTAGTGACCGCCGTACTACTCCTACTCTCGCTACCGGTGCTAGCTGCCGGAATTACCATA $\begin{array}{llllllllllllllllllllllllllllllllllllllll}L & L & T & D & R & N & L & N & T & T & F & F & D & P & A & G & G & G & D & P & I & L & Y & Q & H & L & F & W & F & F & G & H & P & E\end{array}$ 6101 CTACTGACAGATCGAAATCTAAACACAACATTCTTTGACCCTGCTGGAGGAGGAGACCCTATTCTATACCAACACCTATTCTGATTCTTCGGCCATCCTG $\begin{array}{lllllllllllllllllllllllllllllllllll}V & Y & I & L & I & L & P & G & F & G & M & I & S & H & I & V & A & Y & Y & S & G & K & K & E & P & F & G & Y & M & G & M & V & W\end{array}$ 6201 AagTATACATCCTAATTTTACCAGGATTTGGTATAATCTCACACATTGTGGCCTACTACTCTGGAAAGAAAGAACCATTCGGGTATATAGGTATAGTATG $\begin{array}{llllllllllllllllllllllllllllllllllll}A & M & M & A & I & G & L & L & G & F & I & V & W & A & H & H & M & F & T & V & G & M & D & V & D & T & R & A & Y & F & T & S & A\end{array}$

6301 AGCTATAATGGCAATTGGACTTCTAGGCTTCATCGTATGAGCCCATCATATATTTACCGTAGGAATGGATGTTGACACACGAGCATACTTTACATCAGCA $\begin{array}{lllllllllllllllllllllllllllllllllllllllllll}T & M & I & I & A & I & P & T & G & V & K & V & F & S & W & L & A & T & L & G & G & G & V & T & K & W & D & T & P & L & L & W & A & L\end{array}$ 6401 ACCATAATTAITGCCATCCCAACAGGAGTAAAAGTGTTCAGCTGACTAGCGACACTTCACGGAGGAGTGACCAAATGAGACACACCCCTGCTATGAGCAC $\begin{array}{lllllllllllllllllllllllllllllllllll}G & F & I & F & \text { L } & F & T & V & G & G & \text { L } & \text { T } & G & \text { I } & V & \text { L } & A & \text { N } & \text { S } & \text { S } & \text { L } & \text { D } & \text { I } & \text { I } & \text { L } & \text { H } & D & \text { T } & Y & Y & V & V & A\end{array}$ 6501 TAGGATTTATCITTCTTTTTACAGTAGGAGGCCTAACAGGCATCGTACTGGCAAACTCATCACTAGACATCATCCTACATGACACTTATTACGTAGTAGC $\begin{array}{llllllllllllllllllllllllllllllllllll}H & F & H & Y & V & L & S & M & G & A & V & F & A & I & M & G & G & L & V & H & W & F & P & I & M & T & G & Y & T & I & H & N & T\end{array}$ 6601 ACACTTCCACTATGTCCTATCAATAGgAGCAGTATTTGCAATCATAGGGGGACTCGTGCACTGATTTCCACTAATAACAGGATATACCTTACACAACACA 


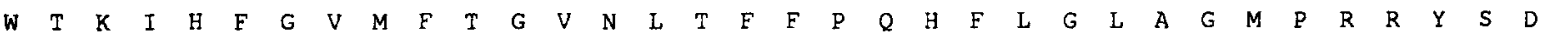
6701 TGAACAAAAATCCACTTTGGTGTAATATTCACAGGAG TAAACCTAACATTTTTCCCACAACACTTCCTCGGACTAGCAGGAATACCACGACGTTACTCAG

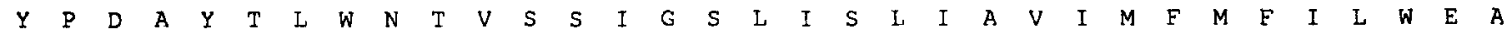
6801 ACTATCCAGATGCCTATACTTTATGAAACACAGTATCATCAATTGGCTCTCTAATITCACTAATTGCCGTAATCATATTTATATTTATCCTGTGAGAAGC $\begin{array}{llllllllllllllllllllllllllllllllllllll}F & S & A & K & R & E & V & L & I & V & E & M & T & T & T & N & V & E & W & L & H & G & C & P & P & P & H & H & T & Y & E & E & P\end{array}$

6901 TTPCTCTGCCAAACGAGAAGTACTAATTGTAGAAATAACAACAACAAATGTAGAATGGCTGCACGGATGCCCACCACCACACCACACATATGAAGAACCA.

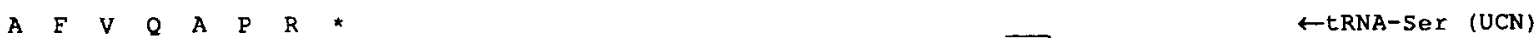

7001 GCATTCGTACAAGCTCCTCGATAAAACACTGAGAAAAGAGGGAATCGAACCCCCGTCAGCTAATTTCAAGACAGCGGCATCACCACTCTGCCATTTTCTI $\operatorname{COII} \rightarrow$

tRNA-Asp $\rightarrow$ M A

7101 TATTAAGATTCTAGTAAAACAAATTACACTACCTTGTCAAGATAGAATTATGAGTGAAAACCTCATGAACCTTGACCAAATGGCACACCCATCACAGTTA

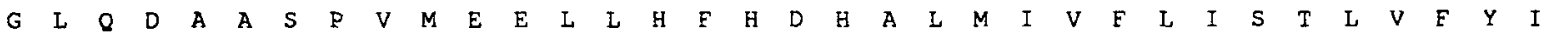
7201 GGATTACAAGATGCAGCTICTCCCGTTATAGAAGAACTCCTCCACPTTCACGATCATGCACTAATAATTGTATTTTPAATTAGCACATTAGTATTTTACA

$\begin{array}{llllllllllllllllllllllllllllllllllll}I & I & A & M & M & T & T & K & M & T & D & K & Y & I & L & D & A & Q & E & I & E & I & V & W & T & L & L & P & A & I & V & L & I\end{array}$

7301 TTATTCTAGCCATAATAACAACAAAAATAACTGACAAATATATCTTAGACGCACAAGAAATTGAAATTGTGTGAACACTACTCCCAGCAATCGTCCTAAT

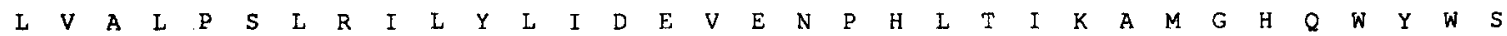

7401 CCTAGTTGCCCTACCCTCGCTACGAATCCTATATCTAATTGATGAAGTCGAAAACCCTCACCTAACAATTAAAGCAATAGGCCACCAATGATACTGAAGC $\begin{array}{lllllllllllllllllllllllllllllllllllllllllll}Y & E & Y & T & D & Y & E & E & L & S & F & D & S & Y & M & T & P & L & Q & D & L & N & P & G & Q & F & R & L & L & E & T & D & H & R\end{array}$

7501 TATGAGTACACGGACTATGAAGAACTAAGCTTCGACTCATACATAACACCACTACAAGACCTAAACCCGGGCCAATTCCGCTTGCTGGAAACAGACCATC

$\begin{array}{llllllllllllllllllllllllllllllllllllllllll}M & V & I & P & M & E & S & L & I & R & V & L & I & S & A & E & D & V & L & H & S & W & A & V & F & A & L & G & V & K & M & D & A\end{array}$

7601 GAATGGTTATCCCAATAGAGTCGCTTATCCGAGTACTAATTTCAGCTGAAGACGTACTACACTCATGAGCAGTCCCAGCCCTAGGAGTAAAAATAGATGC $\begin{array}{lllllllllllllllllllllllllllllllllll}V & P & G & R & L & N & Q & I & T & F & M & I & S & R & P & G & L & Y & Y & G & Q & C & S & E & I & C & G & A & N & H & S & F & M\end{array}$

7701 AGTCCCAGGGCGACTCAACCAAATTACATTCATAATTTCCCGACCAGGACTATATTATGGACAATGCTCAGAGATTTGTGGAGCAAACCACAGCTITATA

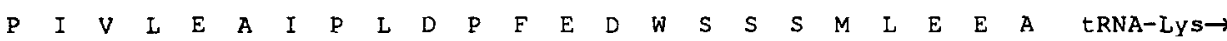

7801 CCCATCGTACTTGAAGCAATCCCACTAGACCCCTTCGAAGACTGATCTTCATCAATGCTGGAAGAAGCCTCACTGAGAAGCTAAATAGAAAGCGATAGCC ATPase $8 \rightarrow$

$\begin{array}{llllllllllllllllllllllllllll}M & P & Q & L & N & P & S & P & W & L & L & I & L & L & E & S & W & L & I\end{array}$

7901 TTTTAAGCTAGAGACTGGTGAAAACAAACCCACCCTCAGTGCCATGCCACAACTAAACCCCTCCCCCTGACTACTAATCCTGCTATTCTCCTGACTCATC $\begin{array}{lllllllllllllllllllllllllllllllllll}F & L & T & M & L & P & S & K & T & Q & L & H & T & F & P & N & M & P & S & T & Q & N & M & C & K & Q & E & P & E & P & W & T & W & P\end{array}$

8001 TTCTTAACTATACTCCCCTCTAAGACACAATTACACACCTTCCCAAACATGCCATCAACACAAAATATATGCAAACAAGAACCAGAACCATGAACCTGAC ATPase $6 \rightarrow$

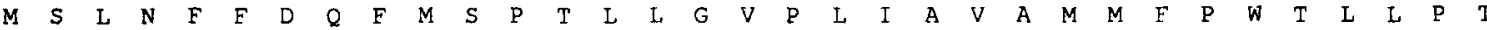
W A *

8101 CATGAGCCTAAACTTCTTTGACCAATTTATGAGCCCAACACTATTAGGAGTACCACTCATTGCTGTAGCAATAATATTCCCATGGACCCTATTACCAACA $\begin{array}{llllllllllllllllllllllllllllllllllll}P & T & N & R & W & L & N & N & R & T & L & T & L & Q & N & W & F & I & G & R & F & T & N & Q & L & L & Q & P & L & N & T & G & G & H\end{array}$

8201 CCAACCAACCGATGACTTAATAACCGAACACTAACACTACAAAACTGATTTATCGGCCGCTTCACTAATCAACTACTACAACCATTAAACACTGGAGGAC $\begin{array}{llllllllllllllllllllllllllllllllllll}K & W & A & M & I & L & M & S & L & N & L & L & G & L & L & P & Y & T & F & T & P & T & T & Q & L & S & L & N & M & G & L & A & I\end{array}$

8301 ACAAATGAGCAATAATCTTAATATCACTAAACCTCCTGGGACTTCTACCGTATACATTCACACCAACAACACAACTATCACTAAACATGGGACTTGCTAT $\begin{array}{llllllllllllllllllllllllllllllllllll}P & F & W & L & A & T & V & L & L & G & L & R & N & Q & P & T & A & A & L & G & H & L & L & P & E & G & T & P & T & L & L & I & P\end{array}$

8401 TCCATTCTGACTAGCAACAGTATTACTGGGACTGCGTAACCAACCCACTGCCGCGCTAGGACACCTTCTCCCAGAAGGAACACCAACCCTGCTAATCCCA $\begin{array}{lllllllllllllllllllllllllllllllllllll}I & L & I & I & I & E & T & I & S & L & L & I & R & P & F & A & L & G & V & R & L & T & A & N & L & T & A & G & H & L & I & M & Q & L\end{array}$

8501 ATCCTAATTATTATTGAAACARTCAGCCTACTTATCCGCCCCTTCGCCCTAGGAGIACGACTAACAGCCAATCTCACAGCAGGCCACCTCCTAATACAAT

$\begin{array}{lllllllllllllllllllllllllllllllllllllllll}I & A & T & A & A & F & V & L & L & P & M & M & P & T & V & A & L & L & T & T & L & V & L & F & L & L & T & L & L & E & I & A & V\end{array}$

8601 TAATTGCTACCGCCGCCTTCGTACTCCTACCTATAATACCAACAGTAGCATTATTAACAACATTAGTCCTATTCCTCCTGACCCTGCTAGAAATTGCCGT

$$
\operatorname{COIII} \rightarrow
$$

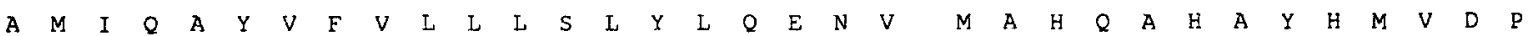

8701 AGCAATAATCCAAGCCTACGTGTTTGTTCTATTACTAAGCCTCTATCTACAAGAAAATGTCTAATGGCCCACCAAGCACACGCATATCACATAGTTGACC

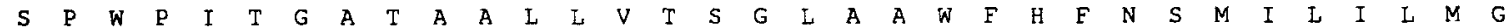

8801 CAAGCCCATGACCCATYACAGGGGCCACGGCCGCCCTACTTGTAACCTCAGGCCTAGCAGCGTGATTTCACTTCAACTCAATAATCTTAATTTTAATAGG

$\begin{array}{lllllllllllllllllllllllllllllllllll} & L & T & L & L & L & L & T & M & Y & Q & W & W & R & D & I & I & R & E & S & T & F & Q & G & H & H & T & L & P & V & Q & K & S & L\end{array}$

8901 ACTAACACTATTGCTACTAACTATGTATCAATGATGACGAGATATTATTCGAGAAAGCACATTCCAAGgTCACCACACACTACCTGTACAAAAAGCCTA $\begin{array}{lllllllllllllllllllllllllllllllllll}R & Y & G & M & I & L & F & I & T & S & E & V & F & F & F & L & G & F & F & W & A & F & Y & \text { H } & \text { S } & S & \text { L } & A & \text { P } & \text { T } & \text { P } & E & \text { L } & G\end{array}$ 9001 CGATATGGTATAATCCTGTTCATTACATCCGAAGTATTCTTCTTCCTAGGGTTCTTCTGAGCCTTTTACCATTCAAGTCTGGCACCCACTCCTGAACTCG $\begin{array}{lllllllllllllllllllllllllllllllllll}G & L & W & P & P & T & G & I & T & P & L & D & P & F & E & V & P & L & L & N & T & A & V & L & L & A & S & G & I & T & V & T & W\end{array}$

9101 GAGGACTCTGACCTCCCACTGGAATTACACCCCTAGATCCATTTGAAGTACCACTATTAAACACAGCAGTTCTACTAGCCTCGGGAATTACAGTCACATG $\begin{array}{lllllllllllllllllllllllllllllllllllll}A & H & H & S & L & M & E & G & Q & R & K & E & A & I & Q & S & L & F & I & T & V & L & L & G & L & Y & F & \text { I } & A & L & Q & A & T\end{array}$

9201 AGCCCATCACAGCCTAATAGAGGGGCAACGAAAAGAGGCTATCCAATCACTATTTATCACAGTTCTGTTAGGACTATACTTTACAGCACTGCAAGCCACA $\begin{array}{lllllllllllllllllllllllllllllllllllllllllllllll}E & Y & Y & E & S & P & F & T & I & A & D & G & A & Y & G & S & T & F & F & V & A & T & G & F & H & G & L & H & V & I & I & G & S & T\end{array}$ 9301 GAATACTACGAATCCCCATTTACAATCGCTGACGGAGCCTATGGCTCAACCTTTTTTGTAGCAACCGGATTCCACGGTCTACATGTCATTATTGGCTCTA $\begin{array}{llllllllllllllllllllllllllllllllllll}F & L & I & V & C & L & V & R & Q & T & Q & Y & \text { H } & F & T & S & N & H & H & F & G & F & E & A & A & A & W & Y & W & H & F & V & D\end{array}$

9401 CATTCCTAATCGTATGCCTAGTACGACAAACACAATACCACTTCACATCAAACCACCACTTTGGCTTTGAAGCAGCAGCATGATACTGACATTTCGTAGA

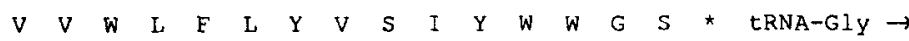

9501 CGTAGTCTGACTATTCTTATACGTATCAATCTACTGATGAGGCTCATAAACCCCTTTAGTACAAAATAATACAAATGACTICCBATCATTTAATCTTGGT

6800

6900

7000

7100

7200

7300

7400

7500

7600

7700

7800

7900

8000

8100

8200

8300

8400

8500

8600

8700

8800

8900

9000

9100

9200

9300

9400

9500

FIGURE 2.-Continued 
NADH $3 \rightarrow$

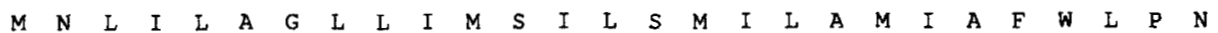

9601 TATACCCCAAGAATGGGTAATGAACCTGATTCTAGCGGGCCTACTTATCATAAGCATCCTCTCTATAATTTTAGCTATAATCGCATTCTGACTACCAAAC $\begin{array}{lllllllllllllllllllllllllllllllllll}M & T & P & D & T & E & K & L & S & P & Y & E & C & G & F & D & P & L & G & S & A & R & L & P & F & S & L & R & F & F & L & V & A & I\end{array}$

9701 ATGACCCCTGATACAGAAAAACTATCTCCCTACGAATGTGGCTTTGATCCTCTAGGATCCGCACGACTCCCATTCTCCCTACGATTTTTCCTAGTAGCAA $\begin{array}{lllllllllllllllllllllllllllllllllll}L & F & L & I & F & D & L & E & I & A & L & L & L & P & L & P & W & A & D & Q & L & T & N & P & T & L & A & L & T & W & T & T & S\end{array}$

9801 TCCTATTCCTGCTATTTGACCTAGAAATTGCATTATTATTACCCCTACCCTGGGCAGACCAACTAACAAACCCAACACTTGCATTAACCTGGACAACAAG

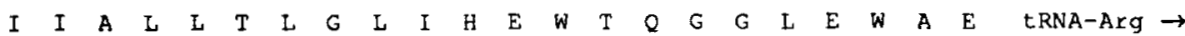

9901 CATCATCGCCCTACTAACACTAGGACTAATCCACGAATGAACTCAAGGAGGCCTCGAATGGGCAGAATAGGTAGTIAGTTTAAAAAAACCACTAATTTCG

$$
\text { NADH } 4 \mathrm{~L} \rightarrow
$$

$\begin{array}{llllllllllllllllllllllllllll}M & T & P & V & Q & L & S & F & N & T & A & F & T & L & G & L & M & G & V & I & F\end{array}$

10001 ACTTAGTTAACTGTGGTGAAACCCCACAACTATCTTCATGACCCCAGTACAACTCAGCTTTAACACTGCATTCACACTAGGCTTAATAGGAGTAACATTC $\begin{array}{lllllllllllllllllllllllllllllllllllll}H & R & A & H & \text { L } & \text { L } & S & A & L & L & C & L & E & G & M & M & L & S & I & Y & M & G & L & S & L & W & P & M & Q & L & E & S & T & T\end{array}$

10101 CACCGAGCCCATCTGCTATCAGCATTACTCTGCCTAGAAGGATAAATATTATCCCTGTATATAGGACTGTCCCTATGACCAATGCAACTAGATCAACTA

$\begin{array}{llllllllllllllllllllllllllllllllll}Y & M & T & T & P & L & L & I & L & A & E & S & A & C & E & A & G & A & G & L & A & L & M & V & A & T & S & R & T & H & G & T & D\end{array}$

10201 CATACATAACCACACCACTACTACTACTCGCCTTCTCAGCCTGTGAGGCTGGAGCAGGCCTAGCCCTCATAGTAGCAACATCCCGCACACATGGTACGGA

$$
\text { NADH } 4 \rightarrow
$$

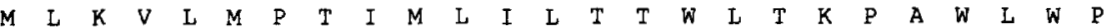

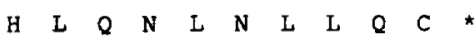

10301 CCACCTCCAAAACCTAAACTTACTACAATGCTAAAAGTTT TAATACCAACAATTATGCTTATCTTAACCACATGATTAACAAAACCTGCATGACTCTGAC

$\begin{array}{lllllllllllllllllllllllllllllllllllll}T & M & T & T & N & S & L & L & V & A & T & I & S & L & T & W & L & K & W & D & S & E & S & G & W & K & S & I & N & S & S & M & A\end{array}$

10401 CAACAATAACAACCAATAGCCTACTCGTAGCTACCATCAGCTTAACCTGACTAAAATGGGACTCAGAGTCAGGATGAAAATCTCTCAACAGCTCAATGGC

$\begin{array}{llllllllllllllllllllllllllllllllllll}T & D & P & L & S & T & P & L & L & I & L & T & C & W & L & L & P & L & M & I & L & A & S & Q & N & H & M & F & M & E & P & L & N\end{array}$

10501 TACCGACCCCCTATCTACACCAT TACTAATCCTCACATGCTGGCTTCTACCCCTCATAATTCTCGCAAGCCAAAACCACATGTTTATAGAACCACTAAAC $\begin{array}{llllllllllllllllllllllllllllllllllllllll}R & Q & R & S & F & I & S & L & L & I & S & L & Q & T & F & L & I & M & A & F & G & A & T & E & I & I & L & F & Y & I & M & F & E & A\end{array}$

10601 CGCCAACGATCATTCATCTCCCTACTCATCTCCCTACAAACATTCCTAATTATAGCATTTGGTGCCACTGAAATCATCCTATTTTACATTATATTIGAAG

$\begin{array}{lllllllllllllllllllllllllllllllllll}T & I & I & P & T & L & I & I & I & T & R & W & G & N & Q & T & E & R & L & N & A & G & T & Y & F & L & F & Y & T & V & M & G & S\end{array}$

10701 CAACCCTAATCCCAACACTAATTATTATTACCCGATGGGGTAICAAACAGAGCGACTAAACGCAGGAACATACTTTTTATTTTATACAGTAATAGGGTC

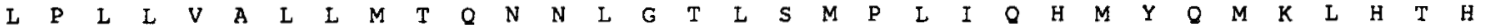

10801 ACTACCACTATTAGTTGCACTTTTAATAACACAAAATAACCTTGGTACCCTATCAATACCGCTCATCCAACACATATACCAAATAAAACTTCATACACAT

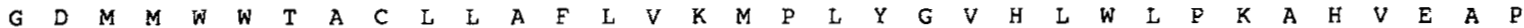

10901 GGAGACATGATATGATGAACAGCCTGCCTATTAGCCTTCTTAGTAAAAATACCACTATACGGAGTCCACCTTTGACTCCCAAAAGCCCATGTAGAAGCCC

I $A \begin{array}{llllllllllllllllllllllllllllllllll} & A & S & M & V & L & A & A & V & I & L & K & L & G & G & Y & G & M & M & R & L & I & M & M & L & A & P & M & T & K & T & L\end{array}$

11001 CAATTGCAGGATCAATAGTACTAGCCGCCGTCCTACTAAAACTAGGAGGATACGGAATAATACGACTAATCATAATATTAGCTCCAATAACAAAAACCCT $\begin{array}{llllllllllllllllllllllllllllllllllll}A & Y & P & F & I & I & L & A & L & W & G & I & I & M & T & G & S & I & C & L & R & Q & T & D & L & K & S & L & I & A & Y & S & S\end{array}$

11101 AGCCTATCCATTCATCATCCTCGCCCTATGAGGATTCATTATAACTGGATCAATCTGCTTACGACAAACAGACCTAAAATCCCTAATCGCCTACTCATCA

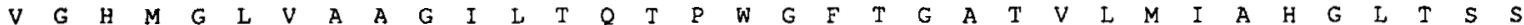

11201 GTAGGCCACATAGGACTAGTGGCAGCAGGTATCCTAACACAAACACCATGAGGCTTTACAGGAGCTACTGTTCTAATAATTGCTCACGGTCTTACATCCT

$\begin{array}{llllllllllllllllllllllllllllllllllll}A & L & F & C & L & A & N & T & N & Y & E & R & T & H & S & R & T & M & I & L & A & R & G & M & O & V & I & L & P & L & M & T & F\end{array}$

11301 CAGCCCTATTCTGTCTAGCAAACACAAACTATGAACGAACCCATAGCCGAACCATGATCCTAGCACGAGGAATGCAAGTTATCCTCCCACTCATGACATI $\begin{array}{lllllllllllllllllllllllllllllllllll}\text { W } & \text { W } & \text { L } & M & \text { M } & \text { N } & \text { L } & \text { A } & \mathbf{N} & \text { L } & \text { A } & \text { L } & \text { P } & \text { P } & \text { S } & \text { T } & \text { N } & \text { L } & \text { M } & \text { G } & \text { E } & \text { L } & \text { L } & \text { I } & \text { I } & \text { T } & \text { M } & \text { T } & F & \text { N } & \text { W } & S & N\end{array}$

11401 CTGATGACTTATAATAAATTTAGCTAACTTAGCCTTACCCCCATCCACTAATCTAATAGGAGAACTACTTATTATTACAATAACCTTCAACTGATCAAAC

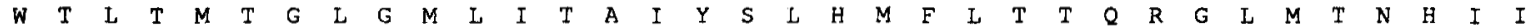

11501 TGGACACTAACTATAACAGGACTGGGCATACTAATCACAGCTATCTACTCATTACACATGTTCCTCACAACACAACGAGGCCTGATAACAAACCACATTA $\begin{array}{llllllllllllllllllllllllllllllllll}S & I & E & P & S & H & T & R & E & H & L & L & M & T & M & H & A & L & P & M & \text { I } & \text { L } & \text { L } & \text { I } & \text { L } & K & \text { P } & E & \text { L } & \text { I } & W & G & W\end{array}$

11601 TCTCAATTGAACCCTCCCACACCCGAGAACACCTACTAATAACAATACATGCCCTCCCAATACTATTGCTAATCCTTAAACCAGAACTGATCTGAGGCTG $S \quad Y \quad$ TRNA-HIS $\rightarrow$ tRNA-Ser (AGY) $\rightarrow$

11701 ATCCTACTGTAAATATAGTTTGAAAAAAACATTAGACTGTGGCTCTAAAAATAAGAGTTAAATCCTCTITATTAACCGAGAGGGGCTAGAGCACTACGAA tRNA-LeU (CUN) $\rightarrow$

11801 GTGGTAATTCTGTAGTACCATGGTTCAATCCCATGGCCCACTCAGCCCCTAAAGGATAATAGCTCATCCATTGGTCTTAGGAGCCAAAAACTCTTGGTGC NADH $5 \rightarrow$

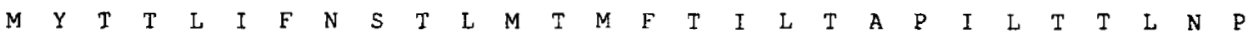

11901 AACTCCAAGTAGCGGCCATGTACACAACATTAATTTTTAACTCAACACTTATAACCATATTTACCATCCTAACAGCCCCAATCTTAACCACCCTTAACCC

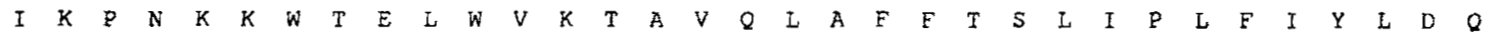

12001 TATCAAACCCAACAAAAAGTGAACAGAATTATGAGTAAAAACCGCGTACAGCTAGCCTTCTTCACAAGTCTAATCCCGCTCTTCATCTATCTTGACCAA $\begin{array}{lllllllllllllllllllllllllllllllllllll}G & I & E & T & I & T & T & N & W & Q & W & M & N & T & N & T & F & N & I & N & I & S & F & K & F & D & Q & Y & S & I & V & F & I & P\end{array}$

12101 GGAATCGAGACCATIACAACAAACTGACAATGAATAAACACCAACACATTTAACATTAACAICAGCTTTAAATTTGACCAATACTCAATTGTATTCATCC $\begin{array}{lllllllllllllllllllllllllllllllllll}I & A & L & Y & V & T & W & S & I & L & E & F & A & N & W & Y & M & H & Q & D & P & K & M & N & Q & F & F & K & Y & L & L & L & F\end{array}$

12201 CAATCGCACTGTATGTCACATGATCAATTCTAGAATTCGCCAACTGATACATGCACCAAGACCCAAAAATAAACCAATTTTTCAAATACCTGCTCCTATT

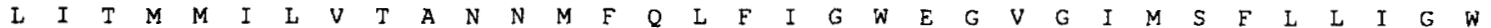

12301 CCTAATCACCATAATAATCTTAGTTACAGCAAACAACATGTTCCAACTATTTATCGGCTGAGAAGGAGTAGGAATTATATCATTCCTATTAATTGGCTGA

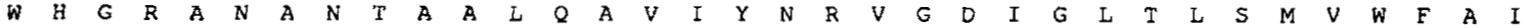
12401 IGACATGGCCGAGCTAACGCCAATACCGCAGCCCTACAAGCAGTCATCTACAATCGAGTAGGAGACATTGGGTIAACCCTAAGCATGGTCTGATTTGCAA

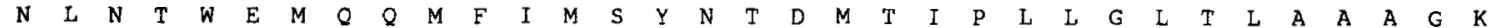
12501 TCAACCTAAATACATGAGAAATACAACAAATATTTATCATATCCTACAACACCGACATAACCATCCCCCTACTAGGCCTAACTCTGGCAGCAGCAGGAAA 


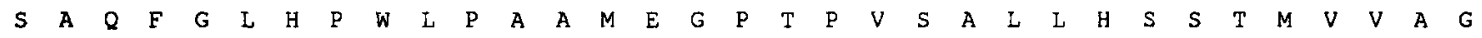

12601 ATCAGCCCAATTGGATtACACCCATGACTACCAGCAGCTATAGAAGGTCCAACACCGGTCTCTGCCCTACTACACTCAAGTACCATAGTGGTTGCCGGA

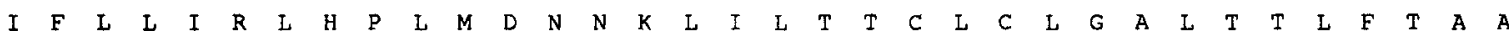

12701 ATCTTCCTGCTTATCCGACTACACCCCCTCATAGACAATAATAAACTAATCCTCACTACCTGCCTCTGCCTAGGAGCACTAACCACCCTATTTACTGCCG

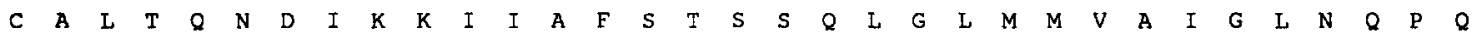

12801 CATGCGCACTCACCCAAAACGATATTAAAAAAATTATTGCATTTTCAACATCAAGTCAACTAGGACTAATAATGGTAGCAATTGGACTAAATCAACCACA

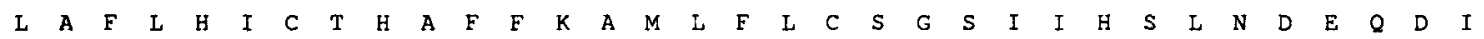

12901 ACTAGCATTCCTCCACATCTGCACCCACGCTTTCTTTAAAGCAATGCTGTTCCTATGCTCCGGATCAATTATTCACAGTCTAAATGATGAACAAGATATC

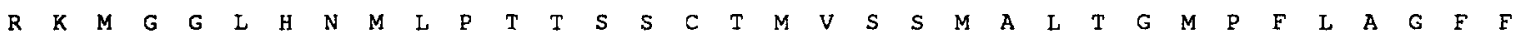

13001 CGAAAAATAGGTGGCTTACACAACATGCTCCCAACAACAAGCTCCTGTACAATAGTTAGCAGCATAGCCCTAACAGGAATGCCATTCCTAGCAGGCITCI

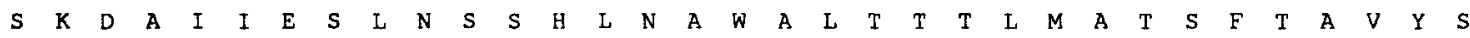

13101 TCTCAAAAGACGCAATCATCGAATCACTAAACTCCTCTCACCTAAACGCCTGAGCCCTAACTACTACACTAATAGCCACATCATTCACCGCAGTCTATAG

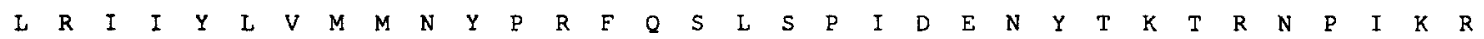

13201 CCTCCGAATTATTTACCTTGTAATGATAAACTATCCACGATTTCAAAGCCTGTCCCCCATCGATGAAAATTATACAAAAACACGTAACCCAATTAAACGA

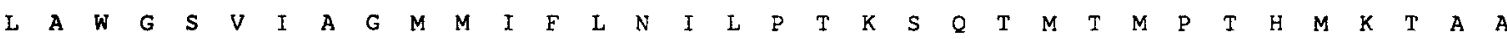

13301 CTAGCATGAGGAAGCGTAATCGCAGGAATAATGATCTTCCTAAACATCCTACCAACTAAATCACAAACAATGACCATACCCACCCATATAAAAACAGCCG

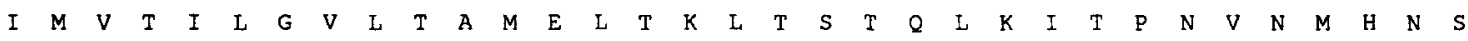

13401 CAATCATAGTTACAATCCTAGGAGTCCTCACCGCTATAGAACTAACAAAACTTACAAGCACACAACTAAAAATCACCCCAAACGTCAACATACACAACTC

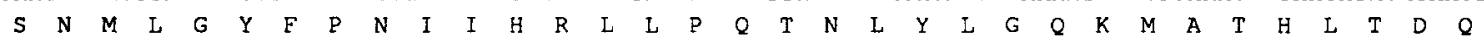

13501 ATCCAACATACTAGgATACTTCCCAAACATCATTCACCGACTTCTACCACAAACAAACTTATATCTAGGACAAAAAATAGCAACACACCTAACAGATCAA

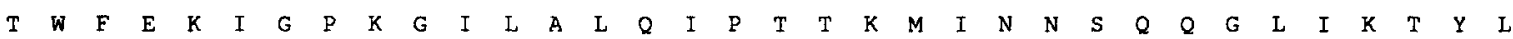

13601 ACATGATTGAAAAAATCGgGCCAAAAGGAATTTTAGCCCTACAAATCCCTACAACTAAAATAATCAACAACTCGCAACAAGGACTAATCAAAACATACC $\begin{array}{lllllllllllllllllllll} & \mathrm{L} & \mathrm{F} & \mathrm{F} & \mathrm{L} & \mathrm{T} & \mathrm{T} & V & \mathrm{~L} & \mathrm{~F} & \mathrm{~T} & \mathrm{~T} & \mathrm{M} & \mathrm{T} & \mathrm{M} & \mathrm{I} & \text { * }\end{array}$

13701 TGACCCTATTTTTCTAACAACAGTTCTATTTACTACAATAACCATAATCTAACTGCTCGAAGCGCACCACGGGACAACCCACGAGTAATCTCCAAAACC

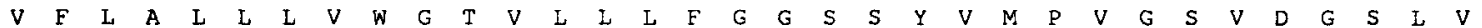

13801 ACAAACAAAGCCAACAATAACACCCACCCAGICACCAACAACAAAAATCCCCCAGAAGAATAAACTATGGGCACACCACTGACATCCCCTGACAAAACTG

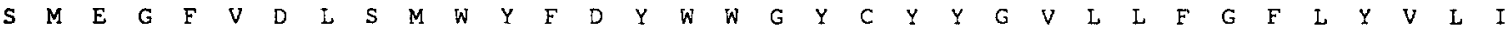

13901 ATATCTCACCAAAAACATCCAATGATATTCAATAAAATTCATACCACCACCCATAACAATAATACCCAACAAGTAAAAAGCCAAACAAATAAACCAAAAT

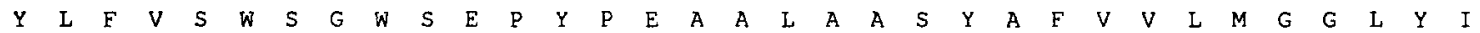

14001 ATAAAGAAAAACCGACCAACTCCCCCAAGACTCAGGATAAGGCTCTGCTGCTAAAGCAGCAGAATAAGCAAAAACAACCAATATCCCCCCCAAATAAATC

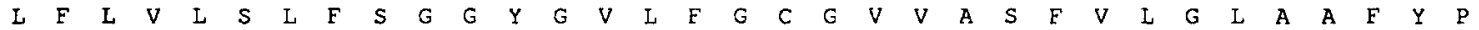

14101 AAAAACAGTACTAAGgACAAAAAAGAGCCACCGTACCCAACCAAAAAGCCACAGCCAACAACTGCAGAAAATACTAACCCAAGAGCAGCAAAATAAGGAG $\leftarrow$ NADH 6

$\begin{array}{lllllllllllllllllllllllll}A & P & N & S & A & V & A & M & L & G & V & V & L & G & M & L & V & V & F & V & F & Y & I & M\end{array}$

14201 CTGGATTAGACGCAACTGCCATCAAACCAACCACGAGTCCCATCAACACAACAAAAACAAAATAAATCATAATTCTTGCCCGGACTTTAACCAGAACCAA Cyt $\mathrm{b} \rightarrow$

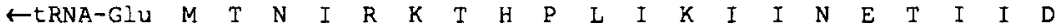

14301 TGACTTGAAAAACCACCGTTGTCAATTCAACTACAAAAACCCATGACAAACATCCGAAAGACACACCCGCTAATTAAAATTATCAACGAAACCATCATCG

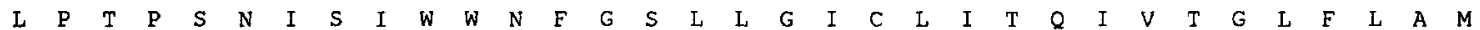

14401 ACCTCCCCACACCATCAAACATCTCAATCTGATGAAATTTTGGGTCACTACTAGGAATTTGTTAATTACACAAATCGTAACAGGCCTATTCTTAGCAAT

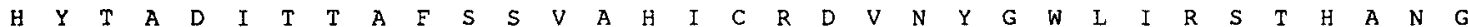

14501 ACACTACACAGCTGACATTACAACAGCATTCTCATCAGTAGCCCACATCTGCCGAGATGTAAACTATGGATGACTAATCCGAAGTACCCATGCCAACGGA

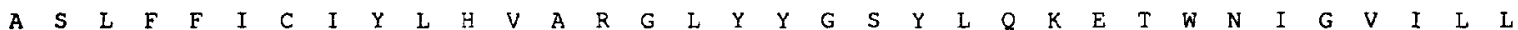
14601 GCCTCTCTATTCTTCATCTGCATCTACCTACATGTAGCACGTGGACTCTACTATGGGTCATACTTACAAAAAGAAACCTGAAACATCGGAGTTATCCTCC

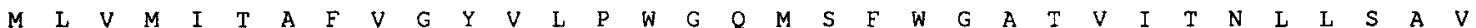

14701 TCATGCTAGTTATGATTACTGCCTTCGTGGGATATGTCCTCCCCTGAGGCCAAATATCATTCTGAGGGGCAACCGTCATCACAAACCTCCTGTCAGCAGT

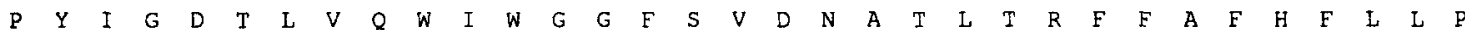

14801 ACCCTATATTGGAGATACACTAGTTCAATGAATCTGAGGAGGTTTTTCCGTAGACAACGCCACACTCACGCGATTCTTTGCCTTCCACTTCCTCCTGCCA

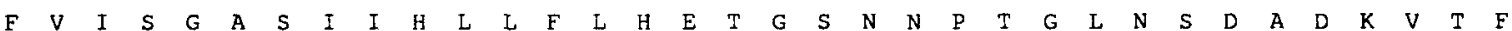
14901 TTCGTAATCTCAGGAGCCTCGATTATTCACCTGCTCTTCCTTCATGAGACAGGATCCAACAACCCAACTGGCCTTAACTCTGACGCAGATAAAGTAACCT

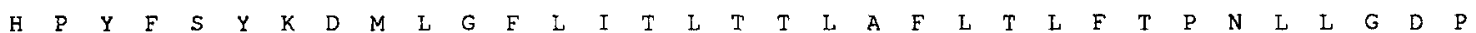

15001 TCCACCCGTACTTCTCGTACAAAGACATGTTAGGATTCCTAATTACACTAACAACACTAGCATTCCTAACCCTATTCACTCCAAACCTGTTAGGAGACCC

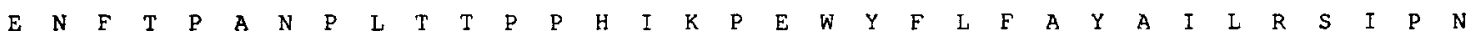

15101 CGAAAACTTCACACCAGCAAACCCATTAACCACCCCGCCACACATCAAACCAGAATGATACTTCCTATTCGCCTATGCAATTCTACGATCCATCCCCAAC

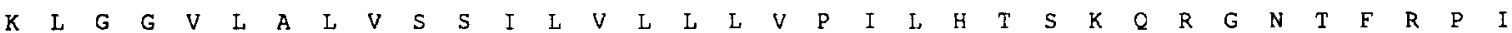
15201 AAACTAGGAGgAGTTCTAGCTCTAGTCTCCTCTATTCTAGTACTACTACTAGTGCCAATCTTACACACCTCAAAACAACGAGGAAACACCTTCCGCCCAA

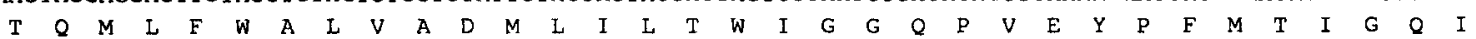

15301 TTACCCAAATACTATTCTGAGCCCTTGTAGCAGACATGCTAATCCTAACCTGAATCGGAGGCCAACCAGTAGAATATCCATTCATGACAATCGGACAAAT $\begin{array}{llllllllllllllllllllllllllllll}A & S & I & T & Y & F & S & L & F & L & I & L & I & P & M & T & G & W & L & E & N & K & A & M & N & W & N & \text { tRNA-Thr } \rightarrow\end{array}$

15401 TGCTTCAATCACCTACTTCAGCCTATTCCTCATCCTAATCCCAATAACTGGATGACTAGAAAACAAGGCCATAAACTGAAACTAGCCTTAGTAGCTTAAA 
15801 ATTAATTACGATGTGGTTAGACACTTATTTCTTAATCTAAACTTGGCTTTATCCATTACTGGCCACTGGTACTGTGCGATGGAGAATAATAGAAAGATT

15901 TATATGATTATAAATTATCTATTACTGGCATCTGGTTTTGGGTTTAGTGAGgGgAaggGCTTTTTAACCCGTAACTCAGTATCACTTTACTATACTGGCC

16101 TTTGGATCTATGACAAAGGATAATCAGTTAATGATAGATAGACATATAATGAATGATAGATAGACATATAATGATGATAAATAGACATATAATGAATGA

16201 TAGTTAGATATATAGATTAATGTAAgAAAgATAATTGAACCCATGACAGAGgACATACTTTTAATGATTTCAGGACATAAACACCATGCACATCAGATTG

16401 ATGTAAT 16407

FIGURE 2.-Continued

phological and molecular characters that could have evolved at the time of this rapid radiation have been obliterated by subsequent changes and back mutations during the last 400 million years. Based on the currently available data, it had remained difficult to distinguish between competing phylogenetic hypotheses, and larger molecular data sets are needed to address this important phylogenetic issue.

Mitochondrial DNA has been widely and successfully used in the past to infer phylogenetic relationships among many different species and might therefore be expected to be a suitable candidate for the evolutionary question at hand. However, several authors (e.g., CumMINGS et al. 1995; RUSSO et al. 1996; ZARDOYA and MEYER 1996c) recently pointed out the high risk of not recovering the exact evolutionary relationships of the taxa under study when individual mitochondrial genes are analyzed and have suggested that larger or more heterogeneous data sets, i.e., complete mitochondrial genomes, are needed to confidently resolve some phylogenetic questions. To address the question of the relationships among living sarcopterygians and to study the evolution of the mitochondrial genome in vertebrates, we previously sequenced the complete mitochondrial genome of an African lungfish (ZARDOYA and MEYER 1996a). Here, we present the complete nucleotide sequence of the mitochondrial genome of the coelacanth, L. chalumnae.

\section{MATERIALS AND METHODS}

DNA extraction, PCR amplification, cloning and sequencing: The scarcity of coelacanth samples (BRUTON and CoUTOUVIDIS 1991; FRICKE 1992; FRICKE et al. 1995) and their generally poor quality did not allow us to isolate mitochondria directly from tissue and to obtain intact mitochondrial genomes. Therefore, a total DNA extraction was performed (TowNER 1991) and the isolated DNA was cleaned through a Sephadex G-50 column. A combination of 24 sets of versatile primers was designed (Table 1) based on highly conserved vertebrate mtDNA regions. They were used to amplify via PCR, contiguous and overlapping fragments (averaging $\sim 800$ bp) which covered the entire coelacanth mtDNA molecule (Figure 1). Some of these primers are expected to successfully amplify mitochondrial DNA fragments in other related vertebrate species (e.g., the primer pairs 19-20 and 27-28 of Table
1 have successfully amplified in fishes and amphibians, unpublished data). Thirty-five cycles of PCR (denaturing at $94^{\circ}$ for $60 \mathrm{sec}$, annealing at $45-50^{\circ}$ for $60 \mathrm{sec}$ and extending at $72^{\circ}$ for $60-105 \mathrm{sec}$ ) were performed in $25-\mu \mathrm{l}$ reactions containing $67 \mathrm{~mm}$ Tris-HCl, $\mathrm{pH} 8.3,1.5 \mathrm{mM} \mathrm{MgCl}_{2}, 0.4 \mathrm{~mm}$ of each dNTP, $2.5 \mu \mathrm{M}$ of each primer, template DNA (10-100 ng), and AmpliTaq DNA polymerase (1 unit, Perkin-Elmer-Cetus).

PCR products were cloned using the $\mathrm{PGEM}-\mathrm{T}$ vector (Promega) and sequenced using the FS-Taq Dye Deoxy Terminator cycle-sequencing kit (Applied Biosystems Inc.) with an automated DNA sequencer (Applied Biosystems 373A) as previously described (ZARDOYA and MEYER 1996a). DNA sequences were obtained using both M13 universal sequencing primers, and three specific oligonucleotide primers for those clones containing inserts $>1000$ bp (see Table 1). Typically, only one clone per PCR product was sequenced. No differences were found in any of the overlapping sequences between contiguous clones. The fidelity of Taq polymerase is $\sim 5 \times 10^{-6}$ errors per nucleotide incorporated per cycle (GELFAND and WHITE 1990) (one error in 16,400 nucleotides after 35 cycles). However, recently XU and ARNASON (1996) have reported that the sequence of the gorilla mitochondrial genome obtained by direct isolation from mitochondria differed outside the control region by 49 nucleotides with respect to the corresponding mitochondrial genome obtained partially by PCR (HORAI et al., 1995). Obviously, not all these differences are due to Taq polymerase error during amplification but also are due to sequencing errors, so we expect in a more realistic estimate that our sequence might differ maximally (outside the control region) by $<20$ nucleotides with respect to the hypothetical sequence from a genome obtained by direct isolation from mitochondria. Based on these calculations, we can submit that the sequence reported is representative of the coelacanth mitochondrial genome and that, although we recommend traditional methods for obtaining mitochondrial genomes if possible, our PCR approach to obtain the complete mitochondrial genome sequence will be useful for other rare or endangered species.

Molecular and phylogenetic analyses: Sequence data were analyzed with the GCG program package (DEVEREUX et al. 1984), MacClade version 3.06 (MADdISON and MADDISON 1992) and PAUP* version d54 (SWOFFORD 1997). DNA sequences were aligned using CLUSTAL W (THOMPSON et al. 1994) followed by refinement by eye. Ambiguous alignments, mainly in $5^{\prime}$ and $3^{\prime}$ ends of protein-coding genes, in tRNA gene sequences corresponding to the tRNA DHU and $\mathrm{T} \psi \mathrm{C}$ arms, and in several highly variable regions of the rRNA genes, were excluded from the phylogenetic analyses (aligned sequences are available from the authors upon request). Third codon positions were also excluded from all phylogenetic analyses. Transitions and transversions were given equal weight 
TABLE 2

Localization of features in the mitochondrial genome of the coelacanth

\begin{tabular}{|c|c|c|c|c|c|}
\hline \multirow[b]{2}{*}{ Feature } & \multirow[b]{2}{*}{ From } & \multirow[b]{2}{*}{ To } & \multirow[b]{2}{*}{ Size (bp) } & \multicolumn{2}{|c|}{ Codon } \\
\hline & & & & Start & Stop \\
\hline tRNA-Phe & 1 & 69 & 69 & & \\
\hline $12 \mathrm{~S}$ rRNA & 70 & 1052 & 983 & & \\
\hline tRNA-Val & 1053 & 1119 & 67 & & \\
\hline 16S rRNA & 1120 & 2784 & 1665 & & \\
\hline tRNA-Leu (UUR) & 2785 & 2860 & 76 & & \\
\hline NADH 1 & 2861 & 3832 & 972 & ATG & TAA \\
\hline tRNA-Ile & 3833 & 3906 & 74 & & \\
\hline tRNA-Gln & 3974 & 3904 & $69(\mathrm{~L})$ & & \\
\hline tRNA-Met & 3974 & 4043 & 70 & & \\
\hline NADH 2 & 4044 & 5090 & 1047 & ATG & TAA \\
\hline tRNA-Trp & 5093 & 5164 & 72 & & \\
\hline tRNA-Ala & 5235 & 5167 & $67(\mathrm{~L})$ & & \\
\hline tRNA-Asn & 5310 & 5238 & $71(\mathrm{~L})$ & & \\
\hline tRNA-Cys & 5403 & 5338 & $65(\mathrm{~L})$ & & \\
\hline tRNA-Tyr & 5475 & 5404 & $69(\mathrm{~L})$ & & \\
\hline CO I & 5477 & 7024 & 1548 & GTG & TAA \\
\hline tRNA-Ser (UCN) & 7100 & 7030 & $69(\mathrm{~L})$ & & \\
\hline tRNA-Asp & 7105 & 7174 & 70 & & \\
\hline $\mathrm{CO}$ II & 7180 & 7870 & 691 & ATG & T-- \\
\hline tRNA-Lys & 7871 & 7943 & 73 & & \\
\hline ATPase 8 & 7944 & 8111 & 168 & ATG & TAA \\
\hline ATPase 6 & 8102 & 8763 & 662 & ATG & TA- \\
\hline CO III & 8764 & 9549 & 786 & ATG & TAA \\
\hline tRNA-Gly & 9550 & 9619 & 70 & & \\
\hline NADH 3 & 9620 & 9968 & 349 & ATG & T-- \\
\hline tRNA-Arg & 9969 & 10,036 & 68 & & \\
\hline NADH 4L & 10,038 & 10,334 & 297 & ATG & TAA \\
\hline $\mathrm{NADH} 4$ & 10,328 & 11,708 & 1381 & ATG & T-- \\
\hline tRNA-His & 11,709 & 11,777 & 69 & & \\
\hline tRNA-Ser (AGY) & 11,778 & 11,844 & 67 & & \\
\hline tRNA-Leu (CUN) & 11,845 & 11,917 & 73 & & \\
\hline NADH 5 & 11,918 & 13,753 & 1836 & ATG & TAA \\
\hline NADH 6 & 14,270 & 13,750 & $519(\mathrm{~L})$ & ATG & AGA \\
\hline tRNA-Glu & 14,340 & 14,271 & $70(\mathrm{~L})$ & & \\
\hline Cyt $b$ & 14,343 & 15,484 & 1142 & ATG & TA- \\
\hline tRNA-Thr & 15,485 & 15,556 & 72 & & \\
\hline tRNA-Pro & 15,626 & 15,558 & $67(\mathrm{~L})$ & & \\
\hline Control region & 15,627 & 16,407 & 781 & & \\
\hline
\end{tabular}

(for exceptions see below). Overlapping positions (two open reading frames) in several genes (ATPase8/ATPase6, ND4L/ $N D 4, N D 5 / N D 6$ ) were duplicated in the analyses. Gaps resulting from the alignment were treated as missing data. The control region was also excluded from the analyses due to its fast rate of evolution which prevented reliable alignment and made it not appropriate for this phylogenetic question.

Three data sets (protein, rRNA, and tRNA coding genes) were combined and subjected to the maximum-parsimony (MP) method (PAUP version 3.1.1, SWOFFORD 1993; and PAUP* version D54; SWOFFORD 1997), using heuristic searches (TBR branch swapping; MULPARS option in effect) with 10 random stepwise additions of taxa to find the most parsimonious trees. Neighbor-joining (NJ) (SAITOU and NEI 1987) (based on Kimura-corrected distance matrices, jumble option in effect) and maximum likelihood (ML) (transversions were given double the weight of transitions; empirical base frequencies and five random stepwise addition of taxa were used) analyses of the sequences were performed with
PHYLIP (version 3.55) (F84 model, FelsENSTEIN 1989), MOLPHY version 2.2 (ADACHI and HASEgawA 1992), and PAUP* version d54 (SWOFFORD 1997). Robustness of the inferred trees was tested by bootstrapping (FELSENSTEIN 1985) (as implemented in PAUP version 3.1.1., PAUP*, and PHYLIP with 100 pseudoreplications each).

Statistical methods: The statistical confidence of MP analyses was evaluated by calculating the standard deviation of the difference in number of steps between the resulting most parsimonious trees and the two alternative hypotheses using the method of TEMPLETON (1983) as implemented in PHYLIP. Similarly, the statistical confidence of the resulting best tree of the ML analysis with respect to competing hypotheses was assessed by calculating the standard deviation of the difference in log-likelihood between the resulting best tree and the alternative hypotheses using the formula of KISHINO and Hasegawa (1989) as implemented in PHYLIP, PAUP*, and MOLPHY. In both cases, competing trees were declared significantly different if the difference in number of steps or 
TABLE 3

Base composition of vertebrate mitochondrial genomes

\begin{tabular}{|c|c|c|c|c|}
\hline & A & $\mathrm{C}$ & G & $\mathrm{T}$ \\
\hline \multicolumn{5}{|l|}{ Proteins } \\
\hline \multicolumn{5}{|l|}{ Tetrapods } \\
\hline 1 & 30.7 & 25 & 21.2 & 23.1 \\
\hline 2 & 19.3 & 27.1 & 12.4 & 41.2 \\
\hline 3 & 41 & 31.9 & 4.8 & 22.2 \\
\hline Total & 30.3 & 28 & 12.8 & 28.9 \\
\hline \multicolumn{5}{|l|}{ Lungfish } \\
\hline 1 & 27.6 & 25.4 & 23.6 & 23.4 \\
\hline 2 & 18.4 & 27.2 & 13.3 & 41.1 \\
\hline 3 & 34.7 & 28.5 & 8.4 & 28.4 \\
\hline Total & 26.9 & 27 & 15.1 & 31 \\
\hline \multicolumn{5}{|l|}{ Coelacanth } \\
\hline 1 & 30.2 & 26 & 23.2 & 20.6 \\
\hline 2 & 18.4 & 27.3 & 13.3 & 41 \\
\hline 3 & 47.7 & 28.3 & 6.9 & 17.1 \\
\hline Total & 32.4 & 27.1 & 14.5 & 26 \\
\hline \multicolumn{5}{|l|}{ Teleosts } \\
\hline 1 & 26.2 & 26.6 & 26.2 & 21 \\
\hline 2 & 18.5 & 27.4 & 13.8 & 40.3 \\
\hline 3 & 37.8 & 33.2 & 8.1 & 20.9 \\
\hline Total & 27.5 & 29.1 & 16 & 27.4 \\
\hline \multicolumn{5}{|l|}{ Bichir } \\
\hline 1 & 29.9 & 23.7 & 23 & 23.4 \\
\hline 2 & 18.9 & 27 & 12.8 & 41.3 \\
\hline 3 & 42.7 & 27.8 & 4.4 & 25.1 \\
\hline Total & 30.5 & 26.2 & 13.4 & 29.9 \\
\hline \multicolumn{5}{|l|}{ Lamprey } \\
\hline 1 & 30.4 & 22.9 & 22.6 & 24.1 \\
\hline 2 & 19 & 26.5 & 12.9 & 41.6 \\
\hline 3 & 41.3 & 21.5 & 3.8 & 33.4 \\
\hline Total & 30.2 & 23.7 & 13.1 & 33 \\
\hline \multicolumn{5}{|l|}{ tRNAs } \\
\hline Tetrapods & 31.9 & 18.6 & 19.8 & 29.7 \\
\hline Lungfish & 28.3 & 20.9 & 23.6 & 27.2 \\
\hline Coelacanth & 30 & 20.8 & 21.8 & 27.4 \\
\hline Teleosts & 28.2 & 21.3 & 23.5 & 27 \\
\hline Bichir & 30.7 & 18.8 & 20.8 & 29.7 \\
\hline Lamprey & 30.4 & 18.8 & 20.4 & 30.4 \\
\hline \multicolumn{5}{|l|}{ rRNAs } \\
\hline Tetrapods & 35.4 & 23.7 & 17.7 & 23.2 \\
\hline Lungfish & 33 & 22.7 & 20.1 & 24.2 \\
\hline Coelacanth & 36.7 & 24.9 & 18.6 & 19.8 \\
\hline Teleosts & 33.4 & 25.4 & 21.5 & 19.7 \\
\hline Bichir & 34.5 & 22 & 19.9 & 23.6 \\
\hline Lamprey & 36.1 & 22.6 & 17.7 & 23.6 \\
\hline
\end{tabular}

Tetrapods: human, Blue whale, opossum, chicken, and frog; teleosts: Rainbow trout, carp, and loach.

Pag-likelihoods where found to be $>1.96$ times the standard deviations (FELSENSTEIN 1989).

The complete mtDNA sequence of the coelacanth has been deposited at the EMBL/GenBank data libraries under accession no. U82228.

\section{RESULTS AND DISCUSSION}

Genome organization and base composition: The complete L-strand nucleotide sequence of the coela- canth mitochondrial genome $(16,407 \mathrm{bp})$ is depicted in Figure 2. The organization of the coelacanth mitochondrial genome conforms to the consensus vertebrate mitochondrial gene order (Figures 1 and 2, Table 2). As in other vertebrates, two rRNAs, 22 tRNAs and 13 proteins are encoded by the coelacanth mitochondrial genome. The overall base composition of the L strand is $\mathrm{A}: 34 \%$; $\mathrm{T}: 24 \%$; $: 27 \%$; and G: $15 \%$. As in other vertebrate mitochondrial genomes, guanine is the rarest nucleotide whereas adenine is the most frequent (MEYER 1993). A more detailed analysis of the base composition was performed by considering the rRNA, the tRNA and the protein coding genes separately (Table 3$)$. In coelacanth protein-coding genes, there is an anti-G bias in third codon positions and pyrimidines are overrepresented in second codon positions, as was noted before for other vertebrate mitochondrial genomes (NAYLOR et al. 1995). Coelacanth tRNAs are A+T rich $(57 \%)$ whereas the rRNAs have a high adenine content (Table 3 ). The noncoding intergenic spacer regions (22 bp), which are likely not subjected to strong selection, showed an $\mathrm{A}+\mathrm{C}$ bias $(73 \%)$. This indicates that, as is the case in other vertebrate mitochondrial genomes, an asymmetrical directional mutation pressure may be operating in the coelacanth genome (JERMIIN et al. 1995).

Noncoding sequences: The control region in the coelacanth mitochondrial genome is $781 \mathrm{bp}$ long and $65 \% \mathrm{~A}+\mathrm{T}$ rich (Figure 2). The coelacanth mitochondrial control region is characterized by the presence of four 22-bp tandem repeats in the right domain, close to the $3^{\prime}$ end. Of these, three are perfect repeats whereas one is imperfect (six nucleotides are different). Even with the presence of these repeats, the coelacanth mitochondrial control region is, so far, the shortest among vertebrates (excluding the atypical control region of the lamprey) (LEE and KOCHER 1995). Neither conserved sequence blocks (CSBs, WALBERG and CLAYTON 1981; SOUTHERN et al. 1988; DILlon and WRIGHT 1993) nor termination associated sequences (TASs, DODA et al. 1981; FORAN et al., 1988), which are commonly found in other vertebrate mitochondrial control regions, could unambiguously be identified in the coelacanth mitochondrial control region. Putative CSB-II and -III motifs, sharing limited sequence similarity to the human and mouse consensus sequence (WALBERG and CLAYTON 1981), were tentatively identified at positions 16,308 and 16,324, respectively (see Figure 2).

The origin of light strand replication $\left(\mathrm{O}_{\mathrm{L}}\right)$ of the coelacanth mitochondrial genome is, as in most vertebrates, located in a cluster of five tRNA genes (WANCY region) (but see SEUTIN et al. 1994) (Figure 2). This region is 26 nucleotides in length and has the potential to fold into a stem-loop secondary structure. The folding of the $\mathrm{O}_{L}$ does not require the use of part of the adjacent tRNA ${ }^{\mathrm{CYS}}$ as has been described for the lungfish mitochondrial genome (ZARDOYA and MEYER 1996a). 
TABLE 4

Amino acid composition of vertebrate mitochondrial proteins

\begin{tabular}{|c|c|c|c|c|c|c|c|}
\hline & Tetrapods & Lungfish & Coelacanth & Teleost & Bichir & Lamprey & Average \\
\hline $\mathrm{A} 1 \mathrm{a}$ & $260(\mathrm{GCC})$ & $312(\mathrm{GCC})$ & $286(\mathrm{GCA})$ & $344(G C C)$ & 307 (GCC) & 297 (GCC) & 301 (GCC) \\
\hline $\mathrm{Arg}$ & 67 (CGA) & 71 (CGA) & $73(\mathrm{CGA})$ & 78 (CGA) & 74 (CGA) & $68(\mathrm{CGA})$ & 72 (CGA) \\
\hline Asn & $151(\mathrm{AAC})$ & 143 (AAY) & $147(\mathrm{AAC})$ & $117(\mathrm{AAC})$ & $147(\mathrm{AAC})$ & $143(\mathrm{AAT})$ & $141(\mathrm{AAC})$ \\
\hline Asp & $67(\mathrm{GAC})$ & 71 (GAC) & $71(\mathrm{GAC})$ & $76(\mathrm{GAC})$ & $73(\mathrm{GAC})$ & 67 (GAC) & 71 (GAC) \\
\hline Cys & 27 (TGC) & 28 (TGY) & 25 (TGC) & 26 (TGC) & 29 (TGC) & $40(\mathrm{TGT})$ & 29 (TGC) \\
\hline Gln & 93 (CAA) & $100(\mathrm{CAA})$ & $103(\mathrm{CAA})$ & $100(\mathrm{CAA})$ & $98(\mathrm{CAA})$ & $98(\mathrm{CAA})$ & 99 (CAA) \\
\hline Glu & 92 (GAA) & 93 (GAA) & $102(\mathrm{GAA})$ & 102 (GAA) & 94 (GAA) & 93 (GAA) & 96 (GAA) \\
\hline Gly & $215(\mathrm{GGA})$ & 246 (GGA) & $239(\mathrm{GGA})$ & 249 (GGA) & $226(\mathrm{GGA})$ & 213 (GGA) & 231 (GGA) \\
\hline $\mathrm{His}$ & $100(\mathrm{CAC})$ & 93 (CAC) & $103(\mathrm{CAC})$ & 105 (CAC) & $98(\mathrm{CAY})$ & $106(\mathrm{CAC})$ & 101 (CAC) \\
\hline Ile & 333 (ATY) & 318 (ATT) & $285(\mathrm{ATC})$ & 281 (ATY) & $356(\mathrm{ATT})$ & 334 (ATT) & 318 (ATT) \\
\hline Leu & $626(\mathrm{CTA})$ & $650(\mathrm{CTW})$ & $622(\mathrm{CTA})$ & 637 (CTA) & $611(\mathrm{CTA})$ & 610 (YTA) & 626 (CTA) \\
\hline Lys & $93(\mathrm{AAA})$ & 72 (AAA) & $84(\mathrm{AAA})$ & $70(\mathrm{AAA})$ & $82(\mathrm{AAA})$ & $99(\mathrm{AAA})$ & 83 (AAA) \\
\hline Met & $210(\mathrm{ATA})$ & 176 (ATA) & 224 (ATA) & 164 (ATA) & $212(\mathrm{ATA})$ & 217 (ATA) & $200(\mathrm{ATA})$ \\
\hline Phe & $226(\mathrm{TTC})$ & 242 (TTT) & 214 (TTC) & 225 (TTY) & 214 (TTY) & 235 (TTT) & 223 (TTY) \\
\hline Pro & 211 (CCM) & $212(\mathrm{CCA})$ & $206(\mathrm{CCA})$ & $209(\mathrm{CCM})$ & $203(\mathrm{CCA})$ & $202(\mathrm{CCA})$ & 207 (CCA) \\
\hline Ser & $288(\mathrm{TCM})$ & 258 (TCM) & $226(\mathrm{TCA})$ & 235 (TCM) & $256(\mathrm{TCA})$ & $263(\mathrm{TCW})$ & 254 (TCM) \\
\hline Thr & $326(\mathrm{ACM})$ & 295 (ACA) & $359(\mathrm{ACA})$ & 305 (ACC) & $299(\mathrm{ACA})$ & $306(\mathrm{ACA})$ & 315 (ACA) \\
\hline $\operatorname{Tr} \mathrm{p}$ & $107(\mathrm{TGA})$ & $116(\mathrm{TGA})$ & 121 (TGA) & $120(\mathrm{TGA})$ & $116(\mathrm{TGA})$ & 109 (TGA) & 114 (TGA) \\
\hline Tyr & 125 (TAY) & 120 (TAY) & 117 (TAY) & $114(\mathrm{TAC})$ & 118 (TAY) & $108(\mathrm{TAT})$ & 117 (TAY) \\
\hline $\mathrm{Val}$ & $169(\mathrm{GTA})$ & $172(\mathrm{GTA})$ & $183(\mathrm{GTA})$ & 222 (GTA) & 174 (GTA) & 192 (GTW) & 185 (GTA) \\
\hline Stop & $13(\mathrm{TAA})$ & 13 (TAA) & $13(\mathrm{TAA})$ & 13 (TAA) & $13(\mathrm{TAA})$ & $13(\mathrm{AGA} / \mathrm{TAA})$ & $13(\mathrm{TAA})$ \\
\hline Total & 3799 & 3801 & 3803 & 3792 & 3800 & 3813 & 3801 \\
\hline
\end{tabular}

Tetrapods: human, Blue whale, opossum, chicken, and frog; teleost: Rainbow trout, carp, and loach. The most commonly used codon for each amino acid is shown in parentheses.

The coelacanth $\mathrm{O}_{\mathrm{L}}$ loop, unlike other fish which have a polypyrimidine tract, but similar to tetrapods, contains a stretch of thymines that is needed for the initiation of L-strand synthesis (WONG and ClaYTON 1985).

Coding sequences: The coelacanth $12 S$ and 165 $r R N A$ genes are 983 and 1665 nucleotides long, respectively (Table 2). The secondary structure of both rRNA genes appears to be reasonably conserved in respect to those of other vertebrates. Most of our rRNA gene sequence showed only minor differences $(99.58 \%$ similarity) to that previously reported (HEDGEs et al. 1993) with the exception of the last $332 \mathrm{bp}$ of the $16 \mathrm{~S} r R N A$ gene sequence, which differed by $20 \%$. These $332 \mathrm{bp}$ correspond to the last PCR fragment amplified by HEDGES et al. (1993) and a search in GenBank revealed that the sequence of this PCR fragment had $96.7 \%$ similarity to that sequence of the alligator $16 \mathrm{~S} r R N A$ gene sequence (HEDGES 1994). This and other reported cases of apparently scrambled sequences during data preparation can be recognized and prevented by individually checking PCR amplified fragments for compatibility in recovering congruent phylogenetic trees (see EDWARDS and ARCTANDER, 1997).

All 22 coelacanth tRNA gene sequences can be folded into a canonical cloverleaf secondary structure with the exception of the $\mathrm{RRNA}^{\mathrm{Ser}(\mathrm{AGY})}$ (data not shown). Two mismatched base pairs in the stems of these putative cloverleaf secondary structures were detected on average for each tRNA. The coelacanth tRNAs ranged in size from 65 to 76 nucleotides (Table 2) and showed size variability in their $\mathrm{DHU}$ and $\mathrm{T} \psi \mathrm{C}$ arms when compared with other vertebrate mitochondrial tRNAs.

The coelacanth mitochondrial genome contains 13 protein-coding genes (Figures 1 and 2); in two cases there is a reading-frame overlap on the same strand (ATPases 8 and 6 share 10 nucleotides; ND4L and ND4 overlap by seven nucleotides). All coelacanth mitochondrial protein-coding genes begin with a ATG start codon except $C O I$, which starts with GTG (Table 2). This initiation codon usage is also found in all other fish mitochondrial genomes that have been completely sequenced so far (TzENG et al. 1992; CHANG et al. 1994; LEE and KOCHER 1995; ZARDOYA et al. 1995; JOHANSEN and BAKKE 1996; NOACK et al. 1996; ZARDOYA and MEYER 1996a). Most coelacanth ORFs end with TAA ( $N D 1$, ND2, COI, ATPase 8, COIII, ND4L and ND5), one ends with AGA (ND6), and the rest have incomplete stop codons, either T (COII, ND3, ND4) or TA (Cyt b) (Table 2).

The codon usage of the coelacanth is similar to that of lamprey (LEE and KOCHER 1995), bichir (NOACK et al. 1996), carp, trout and loach (TzENG et al. 1992; CHANG et al. 1994; ZARDOYA et al. 1995), lungfish (ZARDOYA and MEYER 1996a), and tetrapods (e.g., ROE et al. 1985; DESJARDINS and MORAIS 1990; ARNASON and Gullberg 1993; Horai et al. 1995) (Table 4). A total of 3803 amino acids are encoded by the coelacanth mitochondrial genome. As in other vertebrates, the most abundant amino acid residue encoded by the coelacanth mitochondrial genome is leucine, whereas the 

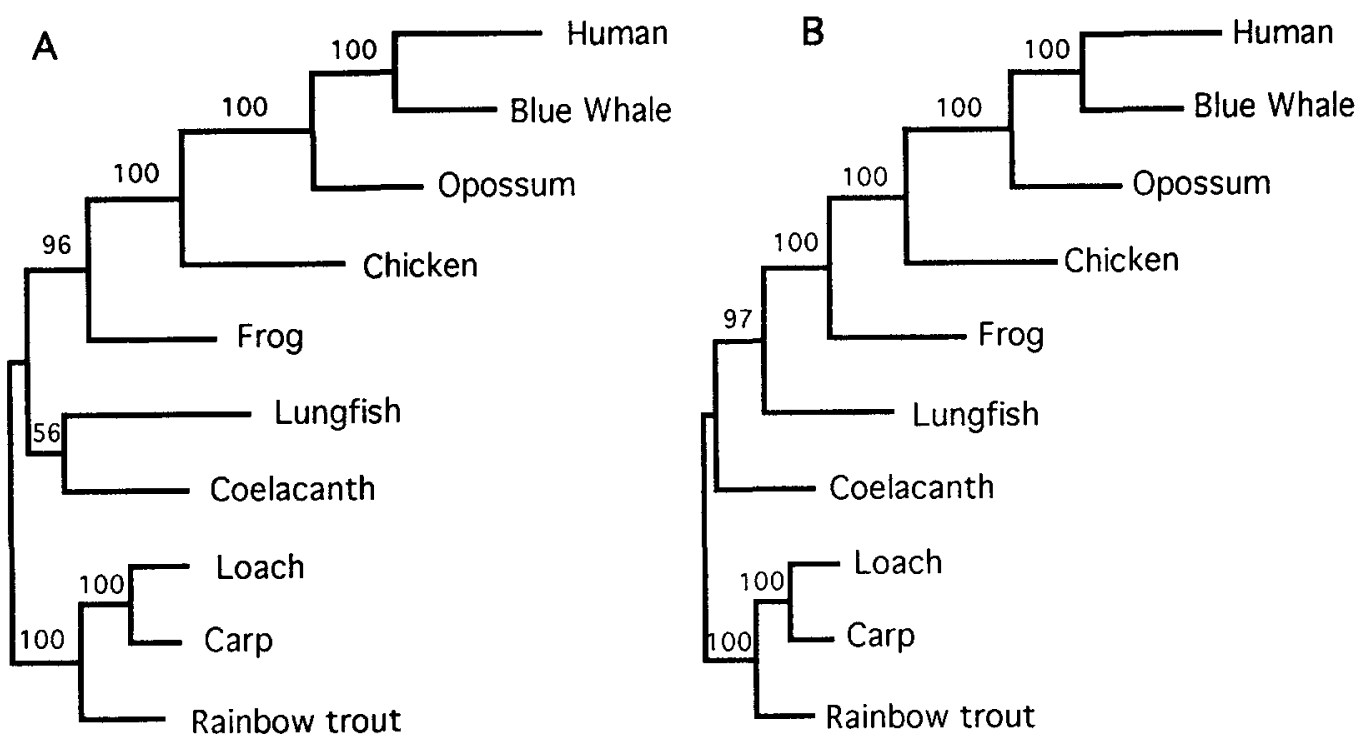

FIGURE 3.-Phylogenetic position of the coelacanth. A data set combining all (protein-coding, rRNA and tRNA) mitochondrial genes was analyzed with maximum parsimony (A), neighbor-joining and maximum likelihood (B) phylogenetic methods. Numbers shown above branches represent bootstrap values from 100 pseudoreplicates for MP (A) and NJ (B). Rainbow trout, carp, and loach were used as outgroup taxa. Third codon positions and transitions in first codon positions of protein coding genes were excluded from the MP phylogenetic analysis. The NJ analysis was performed using Kimura two-parameter distances, an $\alpha$ $=0.53$ (gamma distribution), and excluding third codon positions. The ML analysis was performed with a transition: transversion ratio of 2:1, using the F84 model implemented in PHYLIP version 3.55 (FELSENSTEIN 1989), which accounts for different base frequencies, and excluding third codon positions.

rarest is cysteine (Table 4). Adenines and cytosines are preferentially used in third codon positions of the coelacanth mitochondrial protein coding genes, indicating that codon usage is probably influenced by strand-specific base composition mutational bias (FLOOK et al. 1995; Jermin et al. 1994; LEE and Kocher 1995). Alternatively, third codon position usage may be determined by availability of ribonucleotides in the mitochondria (XIA 1996).

Phylogenetic position of the coelacanth: To asses the position of the coelacanth with respect to lungfishes and tetrapods, a combined data set comprising rRNA, tRNA and protein coding nucleotide sequences of the human (HORAI et al. 1995), blue whale (ARNASON and GULLBERG 1993), opossum (JANKE et al. 1994), chicken (Desjardins and Morais 1990), frog (RoE et al. 1985), carp (ChAng et al. 1994), loach (Tzeng et al. 1992), rainbow trout (ZARDOYA et al. 1995), African lungfish (ZARDOYA and MEYER 1996a), and coelacanth mitochondrial genomes was constructed; it is composed of a total of 16,140 characters. This data set was analyzed with the three most commonly used methods of phylogenetic inference, i.e., maximum parsimony (MP; FITCH 1971), neighbor-joining (NJ; SAITOU and NEI 1987), and maximun likelihood (ML; FELSENSTEIN 1989).

When third codon positions and transitions in first codon positions of the protein-coding gene sequences were excluded from the analysis and teleosts (trout, carp and loach) were included as outgroup taxa, MP recovered one most parsimonious tree (8468 steps, C.I. $=0.65$ ) in which a coelacanth/lungfish clade is identi- fied as the sister group of tetrapods (Figure 3A). This is the same hypothesis as that which is favored by the largest available nuclear DNA data set, the complete $28 \mathrm{~S}$ rRNA gene sequences (ZARDOYA and MEYER 1996b). The node grouping the coelacanth/lungfish clade with tetrapods was supported by a $56 \%$ bootstrap value (Figure 3A). However, if the same data set was analyzed with MP and transitions in first positions of the proteincoding genes were included in the analysis or a transversion:transition weighting of 2:1 was assumed for the whole data set, a lungfish/tetrapod clade was favored with low or moderate bootstrap support (51 and $68 \%$, respectively).

For the NJ analysis, to account for the variation of substitution rates among sites, the $\alpha$ shape parameter of the gamma distribution of rate variation was estimated based on the MP tree (Figure 3A) by the method of YANG and KUMAR (1996). A NJ analysis of the same data set, excluding third codon positions of the proteincoding genes, with teleosts as outgroup taxa, using Kimura 2-parameter distances, and an $\alpha=0.53$, arrived at a tree in which lungfishes are placed as the sister group of tetrapods (97\% bootstrap value for the lungfish+tetrapod node) (Figure 3B). The same topology was recovered when a ML analysis (F84 model, 2:1 transition:transversion ratio; Ln likelihood = $-61,846.08$ ) was performed excluding third codon positions of the protein-coding genes and including teleosts as outgroup taxa. In this case, all branch lengths were found to be significantly greater than zero $(P<$ $0.01)$. 
The disagreement between methods of phylogenetic inference was investigated with KISHINO and HASEGAWA (1989) and Templeton (1983) tests. None of the three possible hypotheses, i.e., lungfish as sister group of tetrapods ( Ln likelihood $=-61,846.08$, best ML tree; 10,812 steps, best MP tree), coelacanth as sister group of tetrapods [Ln likelihood $=-61,863.43, \Delta \mathrm{LnL}=17.35 \pm$ 22.77 (SD); 10,832 steps, $\Delta$ steps $=20 \pm 15.43]$, or coelacanth as sister group of lungfish (Ln likelihood $=$ $-61,859.78, \Delta \mathrm{LnL}=13.70 \pm 22.69 ; 10,813$ steps, $\Delta$ steps $=1 \pm 16.03$ ), could be statistically rejected based on the mitochondrial DNA data set when third codon positions of mitochondrial protein-coding genes were excluded from the analyses and teleosts were used as outgroup taxa. In a separate publication, we will report in more detail on the conflicting phylogenetic signal that is contained in the tRNA, rRNA, and protein mitochondrial data sets and that may be one of the major causes that explain why none of the three hypotheses can be statistically ruled out (R. ZARDOYA, Y. CAO, M. HASEgAwA and A. MeYer, unpublished data).

Our evolutionary analyses confirmed the phylogenetic position of the coelacanth as a closer relative to tetrapods and other sarcopterygians than to the ray-fin fishes (Actinopterygii). These results indicate that the rate of evolution of the mitochondrial genome is appropriate for resolving relationships even among ancient lineages (at least up to the Devonian). However, it seems to fall short of recovering and strongly supporting the relationships among the extant lineage of lobefinned fishes that originated within a narrow window in time $\sim 400$ mya. The rapid origin of the various lobefinned lineages and their rapid radiation before the origin of tetrapods continues to make it difficult to resolve their relationships with confidence.

We thank SCOTT EDWARos and an anonymous reviewer for providing helpful suggestions on the manuscript. The coelacanth tissue was kindly provided by ROBERT MURPHy (Royal Ontario Museum). P.J. PERL assisted in the cloning and automated sequencing. DAVID SwoFFORD kindly granted permission to publish results based on the test version d54 of his PAUP* program. R.Z. was sponsored by a postdoctoral grant of the Ministerio de Educacion y Ciencia of Spain. This work received partial financial support from grants from the National Science Foundation (BSR-9107838, BSR-91 19867, and DEB-9615178) and from a collaboration grant with the Max-Planck-Institut für Biology in Tübingen from the Max-Planck Society, Germany. This publication was prepared during A.M.'s tenure as Miller Visiting Research Professor and a Guggenheim Fellow at the University of California at Berkeley. The support of the John Simon Guggenheim Foundation, and the Miller Institute and the hospitality of the Museum of Vertebrate Zoology and the Departments of Integrative Biology and Molecular and Cell Biology is gratefully acknowledged.

\section{LITERATURE CITED}

ADACHI, J., and M. HASEGAWA, 1992 MOLPHY: Programs for Molecular Phylogenetics I-PROTML: Maximum Likelihood Inference of Protein Phylogeny. Institute of Statistical Mathematics, Tokyo.

Agassiz, L., 1844 Recherches sur les Poissons Fossiles, Imprimerie de Petitpierre, Neuchatel.

AHLBERG, P. E., 1991 A re-examination of sarcopterygian interrela- tionships, with special reference to the porolepiformes. Zool. J. Linn. Soc. 103: 241-288.

Ahlberg, P. E., J. A. ClACK and E. Luksentcs, 1996 Rapid braincase evolution between Panderichthys and the earliest tetrapods. Nature 381: 61-63.

Arnason, U., and A. Gullbers, 1993 Comparison between the complete mtDNA sequences of the blue and the fin whale, two species that can hybridize in nature. J. Mol. Evol. 37: 312-322.

Bemis, W. E., and R. G. NorThcutT, 1991 Innervation of the basicranial muscle of Latimeria chalumnae. Environ. Biol. Fishes 32: $147-158$

Betz, U. A., W. E. MAYER and J. KI.EIN, 1994 Major histocompatibility complex class I genes of the coelacanth Latimeria chalumnae. Proc. Natl. Acad. Sci. USA 91: 11065-11069.

Bruton, M. N., and S. E. Coutouvidis, 1991 An inventory of all known specimens of the coelacanth Latimeria chalumnae, with comments on trends in the catches. Environ. Biol. Fishes 32: $371-390$

CARROL., R. L., 1988 Vertebrate Paleontology and Evolution. Freeman, New York.

ChANG, M. M., 1991 Rhipidistians, pp. 1-28 in Origins of the Higher Croups of Tetrapods. Controversy and Consensus, edited by H.P. Schultze and L. Trueb. Cornell University Press, Ithaca, NY.

Chang, Y. S., F. L. Huang and T. B. Lo, 1994 The complete nucleotide sequence and gene organization of carp (Cyprinus carpio) mitochondrial genome. J. Mol. Evol. 38: 138-155.

Cl.ACK, J. A., 1994 Earliest known tetrapod braincase and the evolution of the stapes and fenestra ovalis. Nature 369: 392-394.

Cloutitr, R., and P. E. AHLberg, 1996 Interrelationships of basal sarcopterygians, pp. 445-479 in Interrelationships of Fishes, edited by M. L. J. Stlassny, L. R. PARenti and G. D. Johnson. Academic Press, San Diego.

Cloutier, R., and P. L. Forey, 1991 Diversity of extinct and living actinistian fishes (Sarcopterygii). Environ. Biol. Fishes 32: 5974.

CourtenaY-Latimer, M., 1979 My story of the first coelacanth. Occas. Pap. Calif. Acad. Sci. 134: 6-10.

Cummings, M. P., S. P. OtTo and J. Wakeley, 1995 Sampling properties of DNA sequence data in phylogenetic analysis. Mol. Biol. Evol. 12: 814-822.

Desjardins, P., and R. Morais, 1990 Sequence and gene organization of the chicken mitochondrial genome. J. Mol. Biol. 212: $599-634$.

Devereux, J., P. HaEberl. and O. Smithies, 1984 A comprehensive set of sequence analysis programs for the VAX. Nucleic Acids Res. 12: 387-395.

Dilion, M. C., and J. M. Wright, 1993 Nucleotide sequence of the D-Loop Region of the sperm whale (Physeter macrocephalus) mitochondrial genome. Mol. Biol. Evol. 10: 296-305.

DODA, J. N., C. T. WRIGHT and D. A. CLAYTON, 1981 Elongation of displacement loop strands in human and mouse mitochondrial DNA is arrested near specific template sequences. Proc. Natl. Acad. Sci. USA 78: 6116-6120.

EDWARDS, S. V., and P. ARGTANDER, 1997 Congruence and phylogenetic re-analysis of perching bird cytochrome $b$ sequences. Mol. Phyl. Evol. 7: 266-271.

Felsenstein, J., 1985 Confidence limits on phylogenies: an approach using the bootstrap. Evolution 39: 783-791.

Felsenstein, J., 1989 PHYLIP-phylogeny inference package (Version 3.4.). Cladistics 5: 164-166.

FiтcH, W.M., 1971 Toward defining the course of evolution: minimal change for a specific tree topology. Syst. Biol. 20: 406-416.

Fiook, P. K., C. H. F. Rowell and G. Gellinsen, 1995 The sequence organization and evolution of the Locusta migratoria mitochondrial genome. J. Mol. Evol. 41: 928-941.

Foran, D. R., J.E. Hixson and W. M. Brown, 1988 Comparisons of ape and human sequences that regulate mitochondrial DNA transcription and D-loop DNA synthesis. Nucleic Acids Res. 16: $5841-5861$.

FORFY, P. L., 1987 Relationships of lungfishes. J. Morphol. 1 Suppl.: $75-91$.

FOREY, P. L., 1988 Golden jubilee for the coelacanth Latimeria chalumnae. Nature 336: 727-732.

FOREY, P. L., 1991 Latimeria chalumnae and its pedigree. Environ. Biol. Fishes 32: $75-97$.

Fricke, H., 1992 Coelacanth tissue bank. Nature 357: 105. 
Fricke, H., K. Hissmann, J. Schauer and R. Plante, 1995 Yet more danger for coelacanths. Nature 374: 314.

FRITZSCH, B., 1987 Inner ear of the coelacanth fish Latimeria has tetrapod affinities. Nature 327: 153-154.

Gelfand, D. H. and WhITE, T.J. 1990 Thermostable DNA polymerases, pp. 129-141 in PCR Protocols: A Guide to Methods and Applications, edited by M. A. INNIS, D. H. GELFAND, J. J. SNISNKY and T. J. WhITE. Academic Press, San Diego.

Gorr, T., T. Kleinschmidt and H. Fricke, 1991 Close tetrapod relationships of the coelacanth Latimeria indicated by haemoglobin sequences. Nature 351: 394-397.

Hedges, S. B., 1994 Molecular evidence for the origin of birds. Proc. Natl. Acad. Sci. USA 91: 2621-2624.

Hedges, S. B., C. A. Hass and L. R. Maxson, 1993 Relations of fish and tetrapods. Nature 363: 501-502.

Hillis, D. M., M. T. DiXon and L. K. AMmerman, 1991 The relationships of the coelacanth Latimeria chalumnae. evidence from sequences of vertebrate $28 \mathrm{~S}$ ribosomal RNA genes. Environ. Biol. Fishes 32: 119-130.

Horal, S., K. Hayasaka, R. Kondo, K. Tsugane and N. Takahata, 1995 Recent African origin of modern humans revealed by complete sequences of hominoid mitochondrial DNAs. Proc. Natl. Acad. Sci. USA 92:532-536.

Huxley, T. H., 1861 Preliminary essay upon the systematic arrangement of the fishes of the Devonian epoch. Figures and descriptions illustrative of British organic remains. Mem. Geol. Surv. U.K. Dec. 10: 1-40.

Janke, A., G. Feidmaier-Fughs, K. Thomas, A. Von Haeseler and S. PAABO, 1994 The marsupial mitochondrial genome and the evolution of placental mammals. Genetics 137: 243-256.

Jermin, L. S., D. Graur, R. M. Lowe and R. H. Crozier, 1994 Analysis of directional mutation pressure and nucleotide content in mitochondrial cytochrome $b$ genes. J. Mol. Evol. 39: 160-173.

JERMIIN, L., D. GRAUR and R. H. CROZIER, 1995 Evidence from analyses of intergenic regions for strand-specific directional mutation pressure in metazoan mtDNA. Mol. Biol. Evol. 12: 558-563.

JOHANSEN, S., and I. BAKKE, 1996 The complete mitochondrial DNA sequence of Atlantic cod (Gadus morhua): relevance to taxonomic studies among codfishes. Mol. Mar. Biol. Biotech. 5: 203-214.

KishinO, H., and M. HASEGaWA, 1989 Evaluation of the maximum likelihood estimate of the evolutionary tree topologies from DNA sequence data, and the branching order in Hominoidea. J. Mol. Evol. 29: 170-179.

Kocher, T. D., W. K. Thomas, A. Meyer, S. V. Edwards, S. Pä́bo $e t$ al. 1989 Dynamics of mitochondrial DNA evolution in animals. Proc. Natl. Acad. Sci. USA 86: 6196-6200.

Kol.B, E., J. I. HARRIS and J. BRIDGEN, 1974 Triose phosphate isomerase EC-5.3.1.1 from the coelacanth: an approach to the rapid determination of an amino-acid sequence with small amounts of material. Biochem. J. 137: 185-197.

LEE, W. J., and T. D. KOCHER, 1995 Complete sequence of a sea lamprey (Petromyzon marinus) mitochondrial genome: early establishment of the vertebrate genome organization. Genetics 139: $873-887$.

LONG, J. A., 1995 The Rise of Fishes: 500 Million Years of Evolution. The John Hopkins University Press, Baltimore.

MADDISON, W. P., and D. R. MADDISON, 1992 MacClade: Analysis of Phylogeny and Character Evolution. Sinauer Associates, Sunderland, MA.

MAISEY, J. G., 1996 Discovering Fossil Fishes. Holt, New York.

MANGUM, C. P., 1991 Urea and chloride sensitivities of coelacanth hemoglobin. Environ. Biol. Fishes 32: 219-222.

MEYER, A., 1993 Evolution of mitochondrial DNA in fishes, pp. 138 in Biochemistry and Molecular Biology of Fishes, edited by P. W. HochachKa and. T. P. MommSen. Elsevier Science, New York.

MEYER, A., 1995 Molecular evidence on the origin of tetrapods and the relationships of the coelacanth. Trends Ecol, Evol. 10: 111116.

MEYER, A., and S. I. DOI.VEN, 1992 Molecules, fossils and the origin of tetrapods. J. Mol. Evol. 35: 102-113.

MeYer, A. A., and A. C. WII SON, 1990 Origin of tetrapods inferred from their mitochondrial DNA affiliation to lungfish. J. Mol. Evol. 31: 359-364

Millor, J., 1954 New facts about coelacanths. Nature 174: 426427.

Millot, J., 1955 The coelacanth. Sci. Am. 193: 34-39.
Naylor, G. J., T. M. Collins and W. M. BRown, 1995 Hydrophobicity and phylogeny. Nature 373: 555-556.

NOACK, K., R. ZARDOYA and A. MEYER, 1996 The complete mitochondrial DNA sequence of the bichir (Polyptemes omatipinnis), a basal ray-finned fish: ancient establishment of the consensus vertebrate gene order. Genetics 144: 1165-1180.

NORTHCUTT, R. G., 1987 Lungfish neural characters and their bearing on sarcopterygian phylogeny. J. Morphol. 1 (Suppl.): 277297.

Palumbi, S.R., A. Martin, S. Romano, W. O. McMillan, L. Stice $e t$ al., 1991 The Simple Fool's Guide to PCR. Department of Zoology, University of Hawaii, Honolulu.

PAnChen, A. L., and T. R. Smithson, 1987 Character diagnosis, fossils and the origin of tetrapods. Biol. Rev. 62: 341-438.

Pechere, J. F., H. Rochat and C. Ferraz, 1978 Parvalbumins from coelacanth muscle. II Amino acid sequence of the two less acidic components. Biochim. Biophys. Acta 536: 269-274.

RoE, B. A., M. Din-Pow, R. K. WII SON and J. F. WONG, 1985 The complete nucleotide sequence of the Xenopus laevis mitochondrial genome. J. Biol. Chem. 260: 9759-9774.

Romer, A. S., 1966 Vertebrate Paleontology. University of Chicago Press, Chicago.

Rosen, D. E., P. L. Forey, B. G. Gardiner and C. Patterson, 198 Lungfishes, tetrapods, paleontolgy, and plesiomorphy. Bull. Am. Natl. Mus. Nat. Hist. 167: 159-276.

Russo, G. A. M., N. T'AKEZAKI and M. NeI, 1996 Efficiencies of different genes and different tree-building methods in recovering a known vertebrate phylogeny. Mol. Biol. Evol. 13: 525-536.

SAITOU, N., and M. NEI, 1987 The neighbor-joining method: a new method for reconstructing phylogenetic trees. Mol. Biol. Evol. 4: 406-425.

Schi.jewen, U., H. Fricke, M. Schartl, J. T. Epplen and S. Pä̈̈o, 1993 Which home for the coelacanth? Nature 363: 406

Schultze, H. P., 1987 Dipnoans as sarcopterygians. J. Morphol. 1 (Suppl.): $39-74$.

Schultze, H. P., 1994 Comparison of hypotheses on the relationships of sarcopterygians. Syst. Biol. 43: 155-173.

Schultze, H. P., and R. Cloutier, 1991 Computed tomography and magnetic resonance imaging studies of Latimeria chalumnae. Environ. Biol. Fishes 32: 159-181.

Seutin, G., B. F. LANG, D. P. Mindei.I. and R. Morais, 1994 Evolution of the WANCY region in amniote mitochondrial DNA. Mol. Biol. Evol. 11: 329-340.

SMITH, J. L. B., 1939 A living fish of mesozoic type. Nature 143: 455456.

SmitH, J. L. B., 1956 Old Fourlegs: The Story of the Coelacanth. Longmans, London.

Southern, S. O., P.J. Southern and A. E. Dizon, 1988 Molecular characterization of a cloned dolphin mitochondrial genome. J Mol. Evol. 28: 32-42.

Stensiō, E. A., 1921 Triassic Fishes from Spizzbergen. Holzhausen, Vienna.

Stock, D. W., K. D. MoberG, L. R. Maxson and G. S. WhitT, 1991 A phylogenetic analysis of the $18 \mathrm{~S}$ ribosomal RNA sequence of coelacanth Latimeria chalumnae. Environ. Biol. Fishes 32: 99-117.

SwOFFORD, D. L., 1993 PAUP: Phylogenetic Analysis Using Parsimony. Illinois Natural History Survey, Champaign, IL.

SwOFFORD, D. L., 1997 PAUP*: Phylogenetic Analysis Using Parsimony (*and other methods), version 4.0. Sinauer Associates, Sunderland, MA.

TAMAI, Y., H. KojImA and K. TAKAYAMA-ABE, 1994 Lipids and myelin proteins in the brains of coelacanth (Latimeria chalumnae), lungfish (Lepidosiren paradoxa and Protopterus aetiopicus), bichir (Polypterus senegalus), and sturgeon (Acipenser ruthenus) (Osteichthyes): phylogenetic implications. Can. J. Fish. Aquat. Sci. 51: $1265-1272$.

Templeton, A. R., 1983 Phylogenetic inference from restriction endonuclease cleavage site maps with particular reference to the evolution of human and the apes. Evolution 37: 221-244.

Thompson, J. D., D. G. Higgins and T. J. GiBson, 1994 CLUSTAL W: improving the sensitivity of progressive multiple sequence alignment through sequence weighting, position specific gap penalties and weight matrix choice. Nucleic Acids Res. 22: 46734680.

Thomson, K. S., 1966 Intercranial mobility in the coelacanth. Science 153: 999-1000. 
Towner, P., 1991 Purification of DNA, pp. 47-68 in Essential Molecular Biology. A Practical Approach, edited by T. A. Brown, Oxford University Press, Oxford.

Tzeng, C. S., C. F. Hui, S. C. Shen and P. C. Huang, 1992 The complete nucleotide sequence of the Crossostoma lacustre mitochondrial genome: conservation and variations among vertebrates. Nucleic Acids Res. 20: 4853-4858.

Vorobyeva, E., and H. P. SCHUlTze, 1991 Description and systematics of panderichthyid fishes with comments on their relationship to tetrapods, pp. 68-109 in Origins of the Major Groups of Tetrapods: Controversies and Consensus, edited by H. P. SCHUlTzE, and L. Trueb, Cornell Univ. Press, Ithaca, NY.

WALBERG, M. W. and D. A. ClaYTon, 1981 Sequence and properties of the human KB cell and mouse L cell D-Loop regions of mitochondrial DNA. Nucleic Acids Res. 9: 5411-5421.

WONG, T. W., and D. A. ClaYTON, 1985 In vitro replication of human mitochondrial DNA: accurate initiation at the origin of light-strand synthesis. Cell 42: 951-958.

WoOdward, A. S., 1891 Catalogue of Fossil Fishes in the British Museum (Natural History). British Museum (Natural History), London.

XIA, X., 1996 Maximizing transcription efficiency causes codon usage bias. Genetics 144: 1309-1320.

$\mathrm{XU}, \mathrm{X}$. and U. ARNASON, 1996 A complete sequence of the mitochondrial genome of the Western lowland gorilla. Mol. Biol. Evol. 13: 691-698.
YANG, Z., and S. KUMAR, 1996 Approximate methods for estimating the pattern of nucleotide substitution and the variation of substitution rates among sites. Mol. Biol. Evol. 13: 650-659.

Yokobori, A. I., M. HaSegawa, T. Ueda, N. Okada, K. Nishikawa et al., 1994 Relationship among coelacanths, lungfishes, and tetrapods: a phylogenetic analysis based on mitochondrial cytochrome oxidase I gene sequences. J. Mol. Evol. 38: 602609.

ZARDOYA, R., and A. MEYER, 1996a The complete nucleotide sequence of the mitochondrial genome of the lungfish (Protopterus dolloi), supports its phylogenetic position as a close relative of land vertebrates. Genetics 142: 1249-1263.

ZARDOYA, R., and A. MEYER, 1996b Evolutionary relationships of the coelacanth, lungfishes, and tetrapods based on the $28 \mathrm{~S}$ ribosomal RNA gene. Proc. Natl. Acad. Sci. USA 93: 5449-5454.

ZARDOYA, R., and A. MEYER, 1996c Phylogenetic performance of mitochondrial protein coding genes in resolving relationships among vertebrates. Mol. Biol. Evol. 13: 933-942.

Zardoya, R., A. Garrido-Pertierra and J. M. Bautista, 1995 The complete nucleotide sequence of the mitochondrial DNA genome of the Rainbow trout, Oncorhynchus mykiss. J. Mol. Evol. 41: 942-951.

Communicating editor: N. TAKAHATA 